\title{
Gradhiva
}

GRADHIV

Revue d'anthropologie et d'histoire des arts

27 | 2018

Sur le vif. Photographie et anthropologie

\section{Dans les archives photographiques de la reconstruction de Beyrouth}

In the Photographic Archives of Beirut's Reconstruction

\section{Sophie Brones}

\section{(2) OpenEdition}

\section{Journals}

Édition électronique

URL : http://journals.openedition.org/gradhiva/3555

DOI : 10.4000/gradhiva.3555

ISSN : 1760-849X

Éditeur

Musée du quai Branly Jacques Chirac

\section{Édition imprimée}

Date de publication : 23 mai 2018

Pagination : 196-225

ISBN : 978-2-35744-110-1

ISSN : 0764-8928

\section{Référence électronique}

Sophie Brones, «Dans les archives photographiques de la reconstruction de Beyrouth », Gradhiva [En ligne], 27 | 2018, mis en ligne le 23 mai 2020, consulté le 07 janvier 2021. URL : http://

journals.openedition.org/gradhiva/3555; DOI : https://doi.org/10.4000/gradhiva.3555

(c) musée du quai Branly 
Dans les archives photographiques
de la reconstruction de Beyrouth

par Sophie Brones

Le contexte des démolitions extensives

qui marquèrent le début de la politique de reconstruction du centre de Beyrouth sous la houlette de la société foncière privée Solidere, à la fin de la guerre civile (1991), voit naître la constitution d'une collection photographique aujourd'hui riche de plusieurs milliers d'images. Cet article rend compte d'une recherche qui traite simultanément des dispositifs de production et d'archivage de la photographie et des représentations elles-mêmes, afin de saisir les écarts entre ce que la photographie donne à voir et ce qu'on lui fait dire. En l'utilisant comme source pour l'écriture d'une nouvelle histoire urbaine, Solidere valorise la photographie documentaire qui s'avère pourtant inefficace pour neutraliser un quartier au passé mouvementé. 
1. Rafic Hariri, entrepreneur libanais ayant fait fortune en Arabie saoudite, revint sur la scène libanaise à la fin de la guerre et parvint à mettre la main sur les opérations de reconstruction du centre-ville en mettant notamment au service de l'administration sa société privée de travaux publics Oger-Liban. Peu après la création de la Société libanaise pour le développement et la reconstruction du centre-ville de Beyrouth (Solidere) en 1991, il fut nommé Premier ministre. Il fut assassiné dans ce même centre-ville en février 2005

2. Le plan établi en 1998 par le géographe allemand Heiko Schmid à partir de photographies aériennes (repris dans Verdeil 2007: 167) donne une précieuse indication sur la chronologie des destructions et des démolitions du centre-ville.

3. J'ai pu mener des enquêtes en 2014 et 2015 grâce au soutien de la Gerda Henkel Stiftung/ LabexMed, A-MidexInitiative d'excellence, Maison méditerranéenne des Sciences de l'homme, Aix-Marseille Université.
En 1991, un an après la fin de la guerre civile (1975-1990), dans le contexte d'un pays fortement divisé, la société foncière privée Solidere - projet de l'ancien Premier ministre Rafic Hariri ${ }^{\mathbf{1}}$ - lança un ambitieux programme de reconstruction du centre partiellement détruit de la capitale libanaise. Afin de transformer l'ancien poumon commercial et cosmopolite de la ville en un nouveau hub régional pour le tourisme et les affaires, Solidere expropria les propriétaires d'appartements et d'immeubles du quartier et fit démolir près de quatre-vingts pour cent du tissu urbain, ce qui généra de vives contestations dans les milieux politiques et au sein de la société civile (Brones 2010 et 2014). Seuls deux cent soixante-cinq bâtiments sur mille immeubles recensés dans le périmètre du projet furent préservés et restaurés (Verdeil 2002: 53) ${ }^{2}$.

En parallèle à ces travaux de démolition, Solidere entreprit de constituer une collection photographique. Cette dernière comprend deux corpus d'images: le premier rassemble essentiellement celles du photographe Mosbah Assi, salarié chez Solidere et chargé de couvrir régulièrement, dès 1993, l'activité de développement et de reconstruction; le second comprend les clichés que des photographes libanais ou étrangers, de renommée internationale, sollicités ponctuellement par Solidere dans le cadre de missions photographiques, ont produits depuis 2008. Cette archive est encore peu connue du public. Solidere la met essentiellement au service de sa politique de communication, publiant les photographies dans ses brochures, rapports annuels et trimestriels à destination de ses clients et actionnaires afin d'illustrer l'actualité de ses projets urbains. Cependant, depuis le milieu des années 2000 , ces photographies sont aussi utilisées en tant que sources historiques dans le cadre de ce qu'il convient d'appeler une «politique culturelle» de la société foncière. Celle-ci se traduit par la constitution d'une collection d'œuvres d'art, l'organisation d'expositions et des publications qui diffusent un discours sur l'histoire de la ville où la photographie ne sert plus seulement d'illustration, mais d'argument et de preuve visuelle du délabrement d'aprèsguerre et de la métamorphose du quartier.

L'enquête dont cet article est issu a débuté en $2014^{3}$, motivée par ce que je percevais au départ comme un paradoxe: la démolition du bâti et la conservation concomitante de ses traces par la photographie. Mais sur ce point, Beyrouth ne fait pas figure d'exception. En revanche la manière dont Solidere a décidé, dans un tel contexte, de classer et d'archiver ses photographies, ainsi que les catégories et les régimes de sens qu'elles recouvrent, mérite toute notre attention.

En combinant l'analyse des images à celle des dispositifs d'archivage - indexations, manipulations, discours et catégorisations -, j'interroge le travail de «documentation photographique» de Mosbah Assi, salarié chez Solidere, et la valorisation d'un «style documentaire», deux catégories définies notamment par Olivier Lugon (2001) dans lesquelles s'inscrivent les deux corpus de l'archive de Solidere. En tant qu'acteur politique, économique et urbain de premier plan, la société foncière dans sa promotion de la photographie est susceptible de marquer l'évolution du statut du médium au Liban. En particulier, la valorisation d'un style documentaire concourt potentiellement à la légitimation de la profession de photographe en tant qu'auteur (dimension peu reconnue aujourd'hui au Liban en dehors des milieux artistiques et des élites transnationales) et à la reconnaissance de sa compétence à renouveler les perceptions d'un territoire. 
Questionnement sur la photographie, donc, mais aussi sur la politique urbaine de Solidere à travers ses pratiques d'archivage. Que dire en effet du pouvoir que lui confère la production de photographies capables de marquer l'imaginaire et les perceptions de Beyrouth, dont une partie, précisément, n'existe plus qu'en images?

\section{Aller aux archives}

Les archives photographiques sont gérées par deux départements au sein de la société, celui de la communication et celui des publications ${ }^{4}$, répartis de part et d'autre d'un palier commun au deuxième étage de l'un des immeubles de Solidere au centre-ville. Des archivistes, un ingénieur spécialiste des réseaux informatiques et un photographe y travaillent quotidiennement. Aucune filière spécifique ni aucun diplôme officiel d'archiviste n'existe actuellement au Liban, mais les professionnels que je rencontre se sont pour la plupart formés au sein de la Fondation arabe pour l'image $\mathbf{5}$ à l'occasion d'un stage ou d'un emploi. Cette association, fondée à Beyrouth en 1997 par des artistes et des photographes, collecte des photographies du monde arabe et des diasporas, et pilote également des projets de formation professionnelle à travers la région.

Le département de communication abrite le bureau personnel de Mosbah Assi, un ensemble de bureaux équipés de scanners, d'ordinateurs, d'étagères et d'armoires où sont conservés les fichiers argentiques, et un espace commun pourvu d'une grande table où l'on m'autorise à consulter les documents. II y a aussi une chambre froide où sont rangés des tirages et l'ensemble des négatifs déjà numérisés (soit une partie seulement des fonds). Le plus souvent, les volets restent fermés afin de filtrer la poussière et la lumière qui nuisent à la conservation du matériel et au travail sur écran. Près de cent mille négatifs y sont conservés (associés à des planches-contact consultables dans des classeurs triés par ordre chronologique depuis 1993), cinq cent trente-cinq tirages ainsi qu'un nombre inconnu de diapositives. À cela s'ajoute depuis 2004 un fonds numérique, dont le nombre exact de clichés, à ce jour non comptabilisé, excède sans doute largement celui des photographies argentiques. Le département des publications est situé de l'autre côté du palier. Ici, point de matériel argentique: c'est essentiellement sur les bases de données informatisées que travaillent les archivistes.

Pour consulter les archives de Solidere encore indisponibles en ligne, j'ai dû remplir un formulaire d'admission, précisant les raisons de ma visite et l'objet de ma recherche. Ce que j'ai fait après avoir été recommandée auprès de la responsable du département de communication par l'une des anciennes directrices de la Fondation arabe pour l'image. Pendant trois semaines, j'ai passé des journées à examiner les archives et à discuter, de façon plus ou moins formelle, avec les archivistes. Afin de me focaliser sur un ensemble à la fois cohérent et limité, j'ai commencé par le matériel le plus ancien conservé au département de communication: les photographies prises entre 1991 (fondation de Solidere) et 2007, dont les planches-contact étaient rangées dans des classeurs. Ce matériel s'avérait par ailleurs plus accessible que les images numériques, car aucun poste informatique n'est mis à disposition du public.
4. Appelés respectivement en anglais, langue officielle de la société foncière, "Communication and Public Relations" et "Corporate Reporting and Publications ", ils gèrent pour l'un la publicité, les relations avec la presse, des événements divers, et pour l'autre l'édition et la recherche, les publications spécialisées et le développement du site Internet.

Pour faciliter la lecture, je les appelle ici «département de communication " et "département des publications ».

5. Voir le site Internet de la Fondation arabe pour l'image: http://www. fai.org.lb/home.aspx" http://www.fai.org.lb/ home.aspx 
6. Album 1 (1993-1994), album 2 (1994), album 3 (1994-1995), album 4 (1995), album 5 (19951996), album 6 (1996), album 7 (1996-1997), album 8 (1997-1998), album 9 (1998-1999), album 10 (1999), album 11 (1999-2000), album 12 (2000-2001), album 13 (2001-2002), album 14 (2002-2004), album 15 (2004-2005-2006-2007).

7. Érigée en 1955, la statue des Martyrs est en fait un groupe statuaire qui représente trois jeunes au moment de leur exécution, hommage aux nationalistes syro-libanais exécutés pendant la Première Guerre mondiale par le pouvoir ottoman. Il fut endommagé pendant la guerre civile et démonté en 1996 afin d'être restauré et remonté à son emplacement d'origine en 2004.

Alors que l'effacement des traces de la guerre a prévalu pour la plupart des immeubles restaurés du centre ville, elles furent ici volontairement conservées.

8. Aucune analyse chiffrée de l'évolution des thèmes n'a été produite dans le cadre de cette première enquête. Toutefois, l'identification de thèmes ou sujets récurrents se fonde sur le constat de leur répétition pour une période historique limitée.

\section{Le théâtre urbain: une représentation avantageuse de la société foncière}

L'inventaire établi au sein de ce département recense sept albums de tirages classés par thème («Exemples d'immeubles à restaurer», «Histoire de la place des Martyrs, 1994 ) ou par périodes («Beyrouth au cours des XIX et $x x^{e}$ siècles », "La vie urbaine avant et pendant la guerre», «Vues aériennes de Beyrouth dans les années 1970», «Guerre et destruction 1976-1992»). Deux autres albums de tirages non numérotés sont consacrés à des objets archéologiques et des maquettes, modèles réduits du centre-ville en cours de reconstruction. Sur chacun des tirages sont indiqués la date de la prise de vue, les dimensions, le sujet de la photographie, sa valeur (tirage original ou retirage) et plus rarement son origine (auteur, institution). Une partie de ces tirages a été acquise auprès d'associations, d'institutions publiques ou de studios privés. Des planches-contact des photographies prises entre 1993 et 2007 sont quant à elles classées dans des albums triés par ordre chronologique ${ }^{6}$. II s'agit essentiellement du travail du photographe Mosbah Assi, hormis quelques clichés attribuables à d'autres photographes indépendants ayant travaillé ponctuellement pour le compte de Solidere.

Les deux ensembles - tirages et planches-contact - donnent à voir des rues et des édifices détruits par la guerre ou en cours de construction, la plupart du temps présentés en séries: immeubles, rues, détails architecturaux et vues aériennes. Dans les albums de planches-contact, des étiquettes indiquent exceptionnellement le nom du secteur et le numéro de la parcelle.

Le premier album contient principalement des images d'édifices détruits et, vers la fin de celui-ci, celles d'événements jugés importants régulièrement représentés au fil des albums. C'est le cas par exemple du démontage de la statue des Martyrs, transportée le 29 mars 1996 (album 6, planche 928). Ce monument situé sur la place des Martyrs - ancien point névralgique de la vie beyrouthine, zone d'intenses combats durant la guerre civile et point de départ de la principale ligne de démarcation de la ville - est un lieu de mémoire et un symbole urbain incontesté ${ }^{7}$. Après 1995, la priorité est donnée aux vues des travaux de restauration et à la diffusion d'une image positive de la société foncière: des écoliers visitent le centre-ville (un enjeu majeur pour des générations qui n'avaient pas connu ce quartier avant la guerre), ainsi que des délégations étrangères (investisseurs canadiens, turcs, égyptiens, etc.), des figures politiques ou des autorités religieuses (Jacques Chirac, des émirs des États du Golfe, un prêtre anglican de Jérusalem, etc.). À partir de 1999, Nasser Chamaa, le président-directeur général de Solidere, apparaît de façon récurrente, en tournée dans le quartier avec des visiteurs ${ }^{8}$. D'autres photographies expriment l'attractivité du centre-ville: un concert de la célèbre chanteuse libanaise Fayrouz (1994), un défilé de mode, un festival de musique, la visite du père Noël, des courses automobiles ou des performances de derviches tourneurs. Sont également fréquentes les images symbolisant le rayonnement et le prestige de la société foncière, celles d'une pratique devenue quasi rituelle: au centre d'information de la société foncière, des visiteurs se regroupent autour de modèles réduits du quartier.

\section{Consulter les fonds: l'expérience du temps}

Un geste simple, feuilleter des classeurs de planches-contact, permet d'entrer physiquement en relation avec une matière brute, déjà organisée mais non encore indexée (Van Lier 1983: 51), une accumulation de photographies 


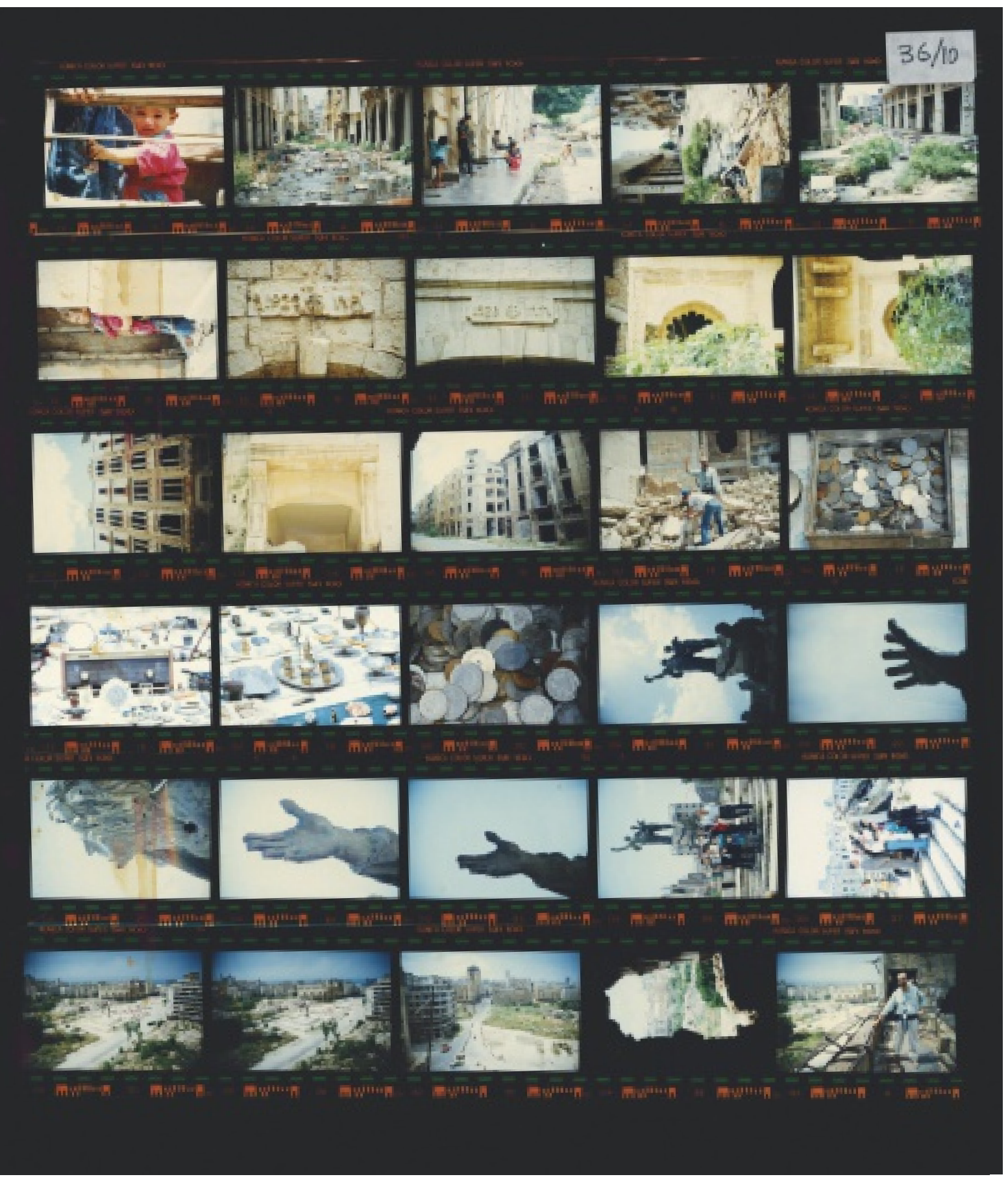

fig. 1

Une planche contact,

sujets divers. Photographies

de Mosbah Assi.

Avec l'aimable autorisation

de Solidere. 
9. Entretien réalisé avec M. Assi, Solidere, janvier 2014.

10. Voir le site Internet de l'artiste:

http://www.fouadelkoury. com/completerrespon. php?theme $=20 \&$ Submit =View des transformations urbaines sur plus de vingt ans. L'écart est d'emblée perceptible entre ce geste et celui consistant à s'asseoir devant un ordinateur pour interroger une base de données dont la matière est soumise à un système d'indexation obéissant à des critères définis a priori par des ingénieurs. Une autre différence fondamentale entre ces deux modes de traitement de la photographie tient à la temporalité: celle de la base informatisée est fragmentée, ne donnant à voir qu'une photographie à la fois, tandis que celle de l'album qui déroule le fil des séries dans une durée est linéaire. Quels peuvent bien être les effets de l'un et l'autre dispositif sur les perceptions du temps et de l'espace représenté?

Feuilleter les albums, c'est faire l'expérience d'un voyage au centre-ville en suivant les itinéraires quotidiens du photographe et la temporalité propre à chaque événement photographié. Celle-ci est reconstruite dans la série, sorte de montage non intentionnel qui permet de restituer chaque image dans son contexte. De ce point de vue, la planche-contact adopte le style du réel: I'hétérogénéité et la juxtaposition d'événements propres à la série photographique rappellent la densité et la synchronie de la vie urbaine (fig. 1).

La planche-contact, dans ses ruptures souvent brutales entre des sujets et des registres d'action que seule une continuité temporelle (celle de l'acte photographique) tient ensemble, rappelle certains livres des surréalistes que Rosalind Krauss, se référant aux photographies de Brassaï et à L'Amour fou d'André Breton, nomme les «Noctambules» (Krauss 1990b). Dans le premier album, par exemple, j'observe tour à tour des vestiges archéologiques, une réception mondaine, des maquettes du quartier et la démolition de la place Ryad-Al-Solh (autre place structurant, avec la place des Martyrs et la place de l'Étoile, un quartier restauré dont l'urbanisme actuel remonte à l'époque du mandat français). Le deuxième album s'ouvre sur des photos du parking de l'ancien complexe du City Center (cinéma et centre commercial à l'architecture emblématique, aujourd'hui encore en ruine aux abords de la place des Martyrs), suivies de celles de la visite du père Noël et des thermes romains. Dans le sixième album, le congrès d'urologie succède aux fouilles du Grand Sérail (palais situé sur la colline du même nom, siège du gouvernement) et aux travaux sur le front de mer! Mais le feuilletage des albums permet aussi de repérer une évolution dans les compétences techniques de l'opérateur. D'abord photographe de presse et reporter au cours de la guerre civile, ce n'est qu'en devenant salarié de la société foncière (en 1993) que Mosbah Assi commence à photographier le paysage urbain et l'architecture ${ }^{9}$. On constate au fil des albums que son regard s'est précisé: il a appris à regarder l'architecture, comme en témoignent des photographies au cadrage plus assuré, dont le propos - un immeuble en ruine, un détail de reconstruction, un événement particulier - est clairement signifié.

Une qualité supplémentaire des planches-contact réside dans la monstration des «temps faibles " de l'enquête photographique (Piette 1992). Par exemple, sur la planche 36 (premier album) on voit plusieurs photos de la main de l'un des personnages de la statue des Martyrs, série qui de toute évidence n'a pas été commandée par la société foncière. Cette digression autour de la main sculptée est une allusion à l'une des photographies les plus connues de Fouad Elkoury, photographe d'origine libanaise, cofondateur de la Fondation arabe pour l'image, intitulée The Hand ${ }^{\mathbf{1 0}}$ (fig. 2). Se révèlent donc ici à la fois l'univers de références du photographe et un imaginaire local en train de se forger. 
II ne fait aucun doute que ces éléments de contexte sont précieux pour l'interprétation des images. Or, une bonne partie de ces données disparait dans l'indexation numérique. Choisir une image, l'extraire d'une série, puis la redocumenter en renseignant les divers champs d'une base de données (date, lieu, contexte, remarques supplémentaires, associations à des catégories, etc.) revient à produire un nouveau discours sur l'image. Interviennent ici les connaissances et plus encore l'expérience mobilisée par les archivistes dans ce travail: pour la plupart très jeunes, ils n'ont pas connu le centre-ville avant le début de la guerre civile (1975), ni même à la fin de celle-ci (1990).

L'expérience beyrouthine pousse en ce sens à son paroxysme l'une des qualités intrinsèques de la photographie, à savoir le principe selon lequel une photographie est toujours à distance du sujet photographié: l'avoir devant nous présuppose nécessairement une coupure temporelle et spatiale d'avec le moment et le sujet de la prise de vue. Quel témoignage l'archive, en tant que système d'indexation, rend-elle possible alors que le matériau convoqué pour "témoigner", en l'occurrence la photographie, est non seulement en lui-même une représentation du réel, celle d'un fragment de réel qui n'existe plus aujourd'hui, mais se trouve de surcroît travaillé par une personne autre que l'opérateur lui-même, ou celui qui l'accompagne?

\section{Manipuler: de la sauvegarde des traces à leur enfouissement}

Le feuilletage des albums de planches-contact conduit aussi à s'interroger sur les pratiques archivistiques, le rapport au document et à la matérialité des photographies (Jungen et Raymond 2012). Les trajectoires et la transformation du document dans l'archive, du négatif au fichier numérique, posent comme on vient de le dire la question de la disparition des éléments de contexte et du sens donné aux images. La chambre froide, étape ultime de l'enfouissement de la trace originale, constitue un "sanctuaire " commun à l'ensemble des fonds des deux départements. Elle est le lieu de stockage des négatifs et tirages, considérés comme des originaux et dont on revendique l'authenticité. Le matériel photographique fait l'objet de précautions importantes: je dois porter des gants pour consulter les albums de planches-contact ou tout autre document, et les négatifs sont nettoyés avant d'être scannés de bout en bout. Tout est numérisé: la première image des films, dont une moitié est totalement claire du fait de l'exposition à la lumière de la pellicule qu'on insère dans l'appareil, mais aussi les photos floues ou les doublons. Que l'image soit visible ou non, il semble donc que les archivistes suspendent temporairement leur jugement quant à la signification et au contenu, chaque millimètre de film, trace tangible de l'activité photographique, vaut en tant que témoignage d'un enregistrement. Les fichiers des photographies numériques ne bénéficient pas du même soin. Le processus d'editing est plus sélectif: seules sont conservées les photos bien cadrées, en bonne définition et dont on comprend au premier abord le sujet. Dans un deuxième temps, toutes les photographies sont temporairement indexées dans la base des archivistes sur le logiciel Adobe Bridge, avec des indications de lieu, de date, des descriptions plus ou moins détaillées de l'image et du contexte, et l'insertion dans une catégorie préétablie comme «restauration" ou "patrimoine", qui correspond aux grands ensembles définis par la société foncière au sein des divisions de l'architecture, de l'urbanisme et du paysage depuis le début des travaux de reconstruction. 


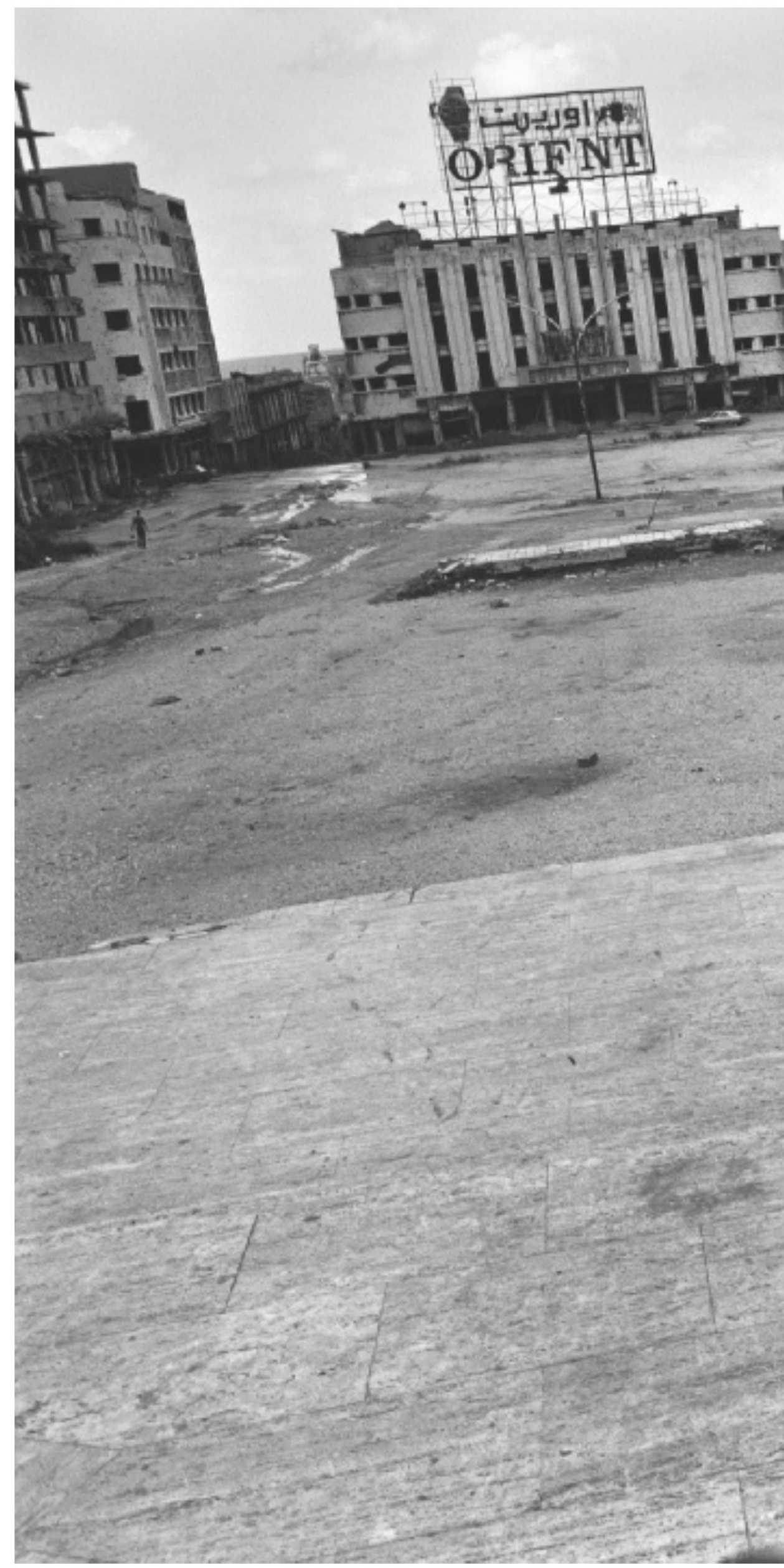




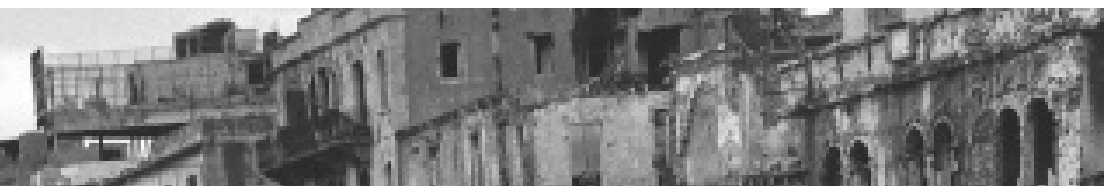

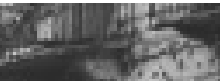

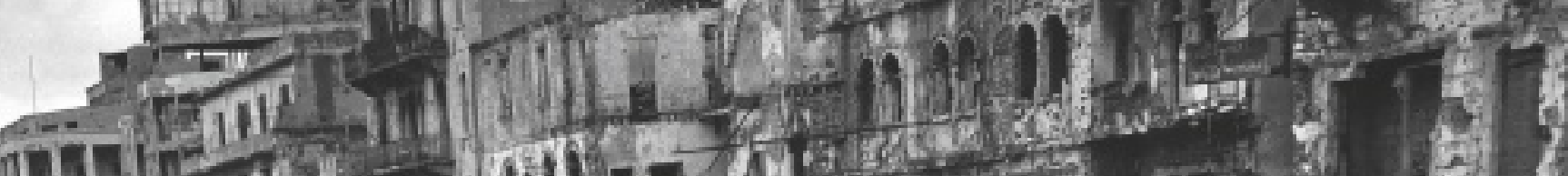

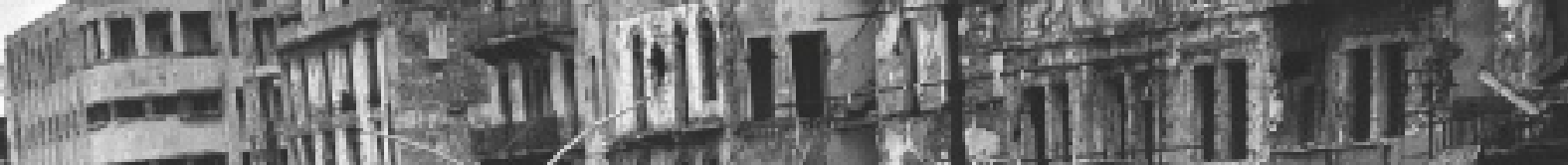

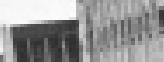

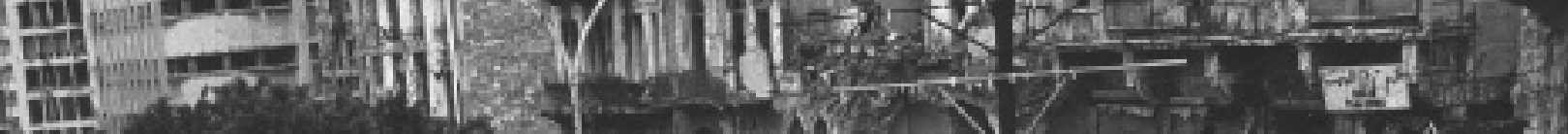

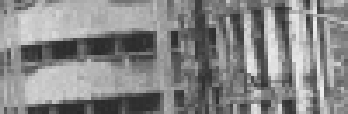

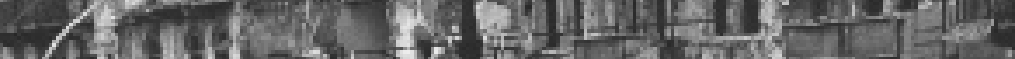
tim.

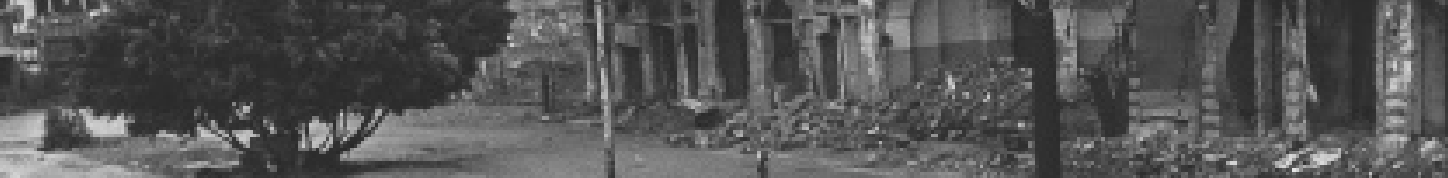

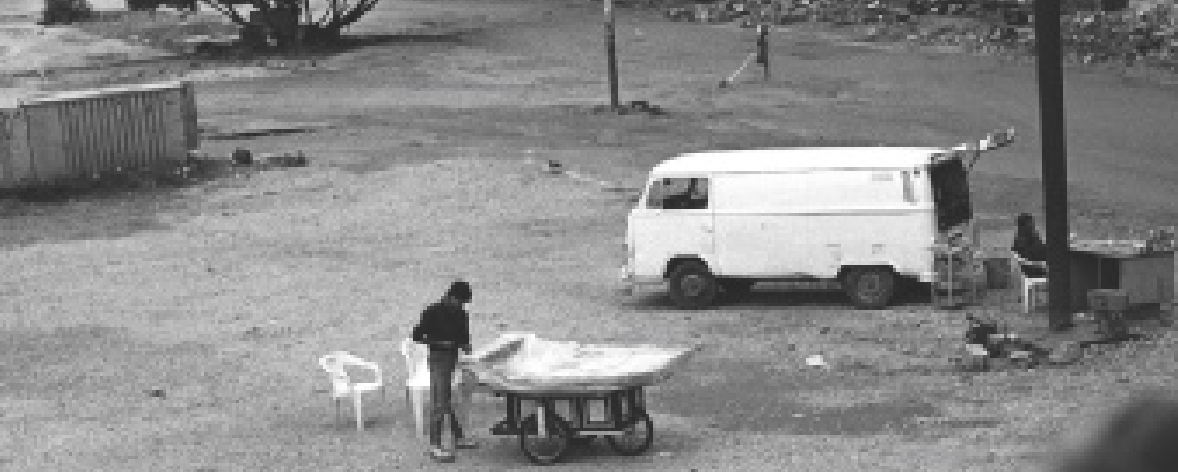


Puis, dans un second temps, après cette première indexation sur Adobe Bridge, l'ensemble des fichiers rejoint la base Cumulus. Ce système, comme la métaphore atmosphérique de son nom l'indique, constitue une sorte de lieu virtuel de la photographie. Près de vingt mille images y étaient stockées en 2016. Les photographies préalablement indexées sur Adobe Bridge y sont réindexées suivant d'autres catégories (par exemple «architecture» ou "souks "). Sans décrire davantage ce système on peut noter que, une fois numérisé, dans la démultiplication de ses occurrences, le négatif voit son rapport au temps et à l'espace radicalement modifié. Extrait de la série, ce « régime de sens " initial qui le connecte à la temporalité particulière de son impression, il est stocké - on pourrait dire enfoui, immobilisé - dans la chambre froide, tandis que l'image numérisée est intégrée à un espace virtuel qui démultiplie ses apparitions sans autre mention du contexte temporel que la date de la prise de vue. La complexité du contexte et des trajectoires de la photographie au sein de l'archive sont ainsi effacées par le processus d'indexation.

\section{Représenter l'architecture}

Si le négatif est traité comme une trace, celle-ci est double: en tant qu'objet, celle d'une pratique photographique, en tant que représentation, celle d'une empreinte lumineuse. La pellicule capte en effet une émanation de la réflexion de la lumière sur des objets en trois dimensions; l'enregistrement puis la révélation produisent l'image en deux dimensions. En cela, la photographie n'adhère pas au réel tel que nous le voyons ou l'expérimentons. Elle en est une représentation (Van Lier 1983; Piette 1992) variant selon la technique de prise de vue (focale, distance, vitesse, profondeur de champ, type de film) et les critères stylistiques retenus (cadrage, lumière, composition, surface, etc.) [About et Chéroux 2001; Shore 2010 (2007)]. Cette «ontologie de la photographie argentique» (Bazin 1999 [1945]) implique donc une réorganisation des éléments du réel dans l'image selon une logique propre à celle-ci, instaurant entre les différents éléments qui la composent un rapport nouveau, spatial, et de sens. Prenons l'exemple de la photographie d'un immeuble en ruine extraite d'une série du département de communication: l'impression de monumentalité de l'édifice ne tient-elle pas moins à l'immeuble lui-même qu'à la façon dont il est cadré, à la distance, au point de vue et au moment choisis par le photographe, quand rien ni personne n'entre dans le cadre (fig. 3)?

Ce constat implique que l'on se prête à une observation critique des images. Prenons un autre exemple, avec la figure ci-dessous (fig. 4) prise dans les premières années d'exercice de Mosbah Assi. Que représente-t-elle? Quelle est son efficacité pour décrire l'architecture? Outre un manque de familiarité de l'opérateur avec le langage de l'architecture et de l'urbanisme, de probables difficultés d'accès aux bâtiments pour des raisons de sécurité, comment expliquer les choix de point de vue, de cadrage ou la distance choisie par le photographe?

Si celle-ci ne permet pas ici de distinguer des détails de l'architecture, le point de vue choisi ne donne à voir qu'un seul aspect d'un immeuble et le cadrage entrave les bâtiments dans leur unité formelle et stylistique. Cette absence apparente de protocole photographique m'est confirmée 


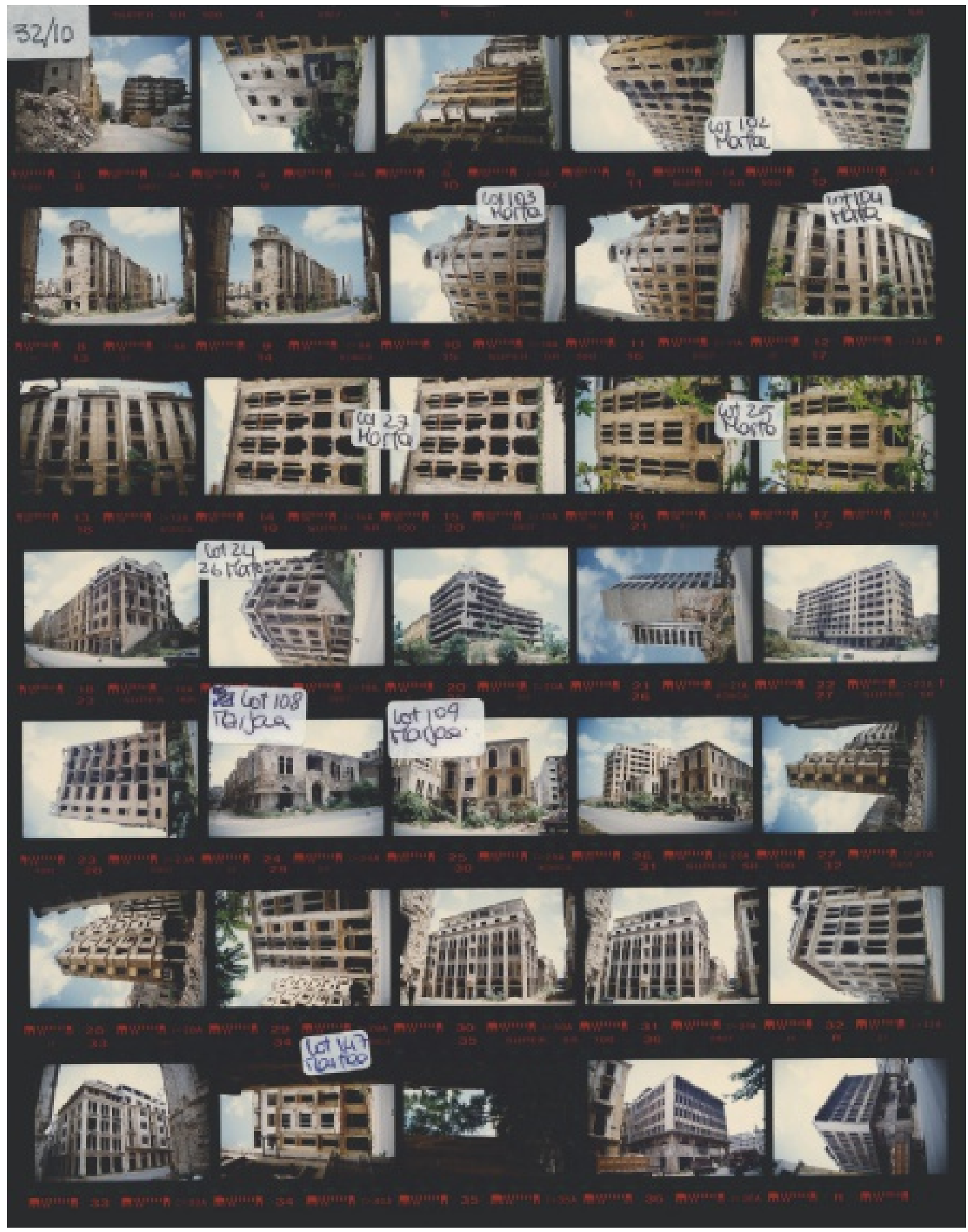

fig. 3 

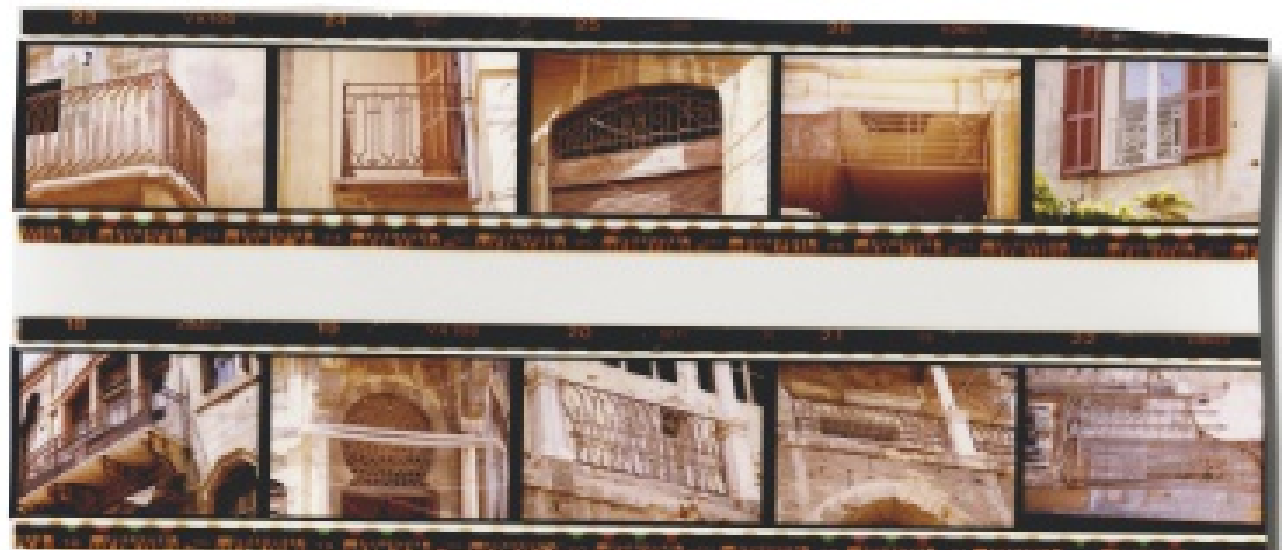

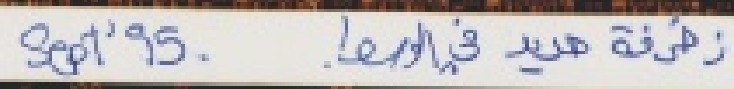
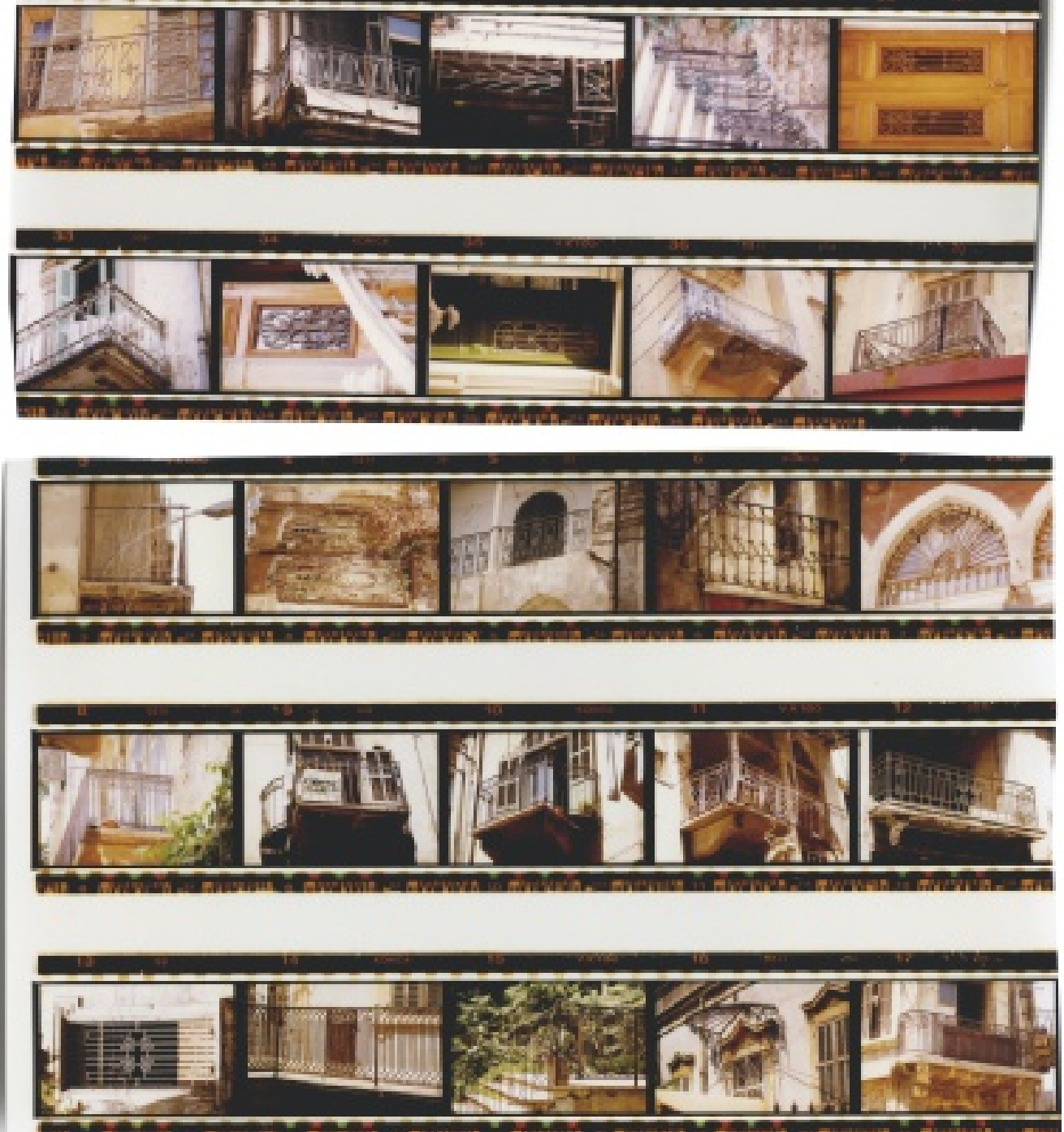

Ror 
par le photographe lui-même: aucune méthode d'inventaire systématique des bâtiments ni aucun protocole de prise de vue n'auraient été mis en place à l'époque ${ }^{11}$.

Comment comprendre la dimension empirique de ces enquêtes photographiques? Dès les premières années de la reconstruction, le photographe dit avoir travaillé de façon à répondre aux demandes des différents départements de «couvrir» des sujets en lien avec l'évolution des projets, la restauration, le développement de nouvelles infrastructures, ou avec des événements participant de l'animation du quartier. De toute évidence, l'importance de l'archive photographique et le prestige qu'elle confère aujourd'hui à la société foncière n'avaient pas été anticipés. Ils ont même sans doute été envisagés assez tardivement, peut-être d'ailleurs seulement à partir de 2008, moment où des missions photographiques ont débuté.

\section{Regards sur la ville: les missions photographiques et l'affirmation d'un style documentaire}

Le département des publications contribue lui aussi à l'enrichissement des collections photographiques de Solidere par l'organisation de missions photographiques. Au début des années 1990, avant d'en léguer une partie au département de communication, il gérait l'intégralité des fonds photographiques. Jusqu'en 2010, il s'occupait aussi de la publication des rapports annuels et semestriels de Solidere. Aujourd'hui, il se charge essentiellement de l'indexation des fonds au sein de la base de données Cumulus et, depuis 2008, de la production de missions photographiques, dont certaines ont donné lieu à des publications (McPherson 2006; Makdissi 2018). Ce département bénéficie donc d'une forme d'antériorité dans la gestion des images photographiques, dont on peut supposer qu'elle participe du léger climat de concurrence perceptible entre les deux départements. Mais on verra plus loin que celui-ci tient sans doute aussi aux qualités attribuées aux images archivées par l'un et par l'autre, ainsi qu'aux deux catégories de photographies qu'ils distinguent. À partir de 2008, les archivistes du département des publications organisèrent donc des missions photographiques afin de documenter l'évolution des travaux de reconstruction. Le photographe Fouad Elkoury en est à l'origine puisque dix ans auparavant, en 1998, il en avait suggéré l'idée à Nohad Makdissi, l'ancienne directrice du département des publications partie à la retraite quelques mois avant le début de mon enquête. Ce projet, la Beirut Central District Photographic Mission, s'inscrivait toutefois dans I'héritage d'une expérience plus ancienne, celle de la Mission photographique de Beyrouth de 1991, dont il convient de dire ici quelques mots.

\section{La Mission photographique de 1991 : un moment fondateur}

Fouad Elkoury, photographe français d'origine libanaise, né en 1952, avait lui-même participé à la Mission photographique de Beyrouth de 1991 aux côtés d'autres photographes jouissant d'une reconnaissance internationale, tels que I'Italien Gabriele Basilico (1944-2013), I'Américain Robert Frank (né en 1924), le Français Raymond Depardon (né en 1942), le Tchèque Josef Koudelka (né en 1938) et le Suisse René Burri (1933-2014).
11. En effet, nombre d'entreprises comparables dans leur intention documentaire reposent sur un protocole de prise de vue, que ce soit chez Bernd et Hilla Becher, Gabriele Basilico ou bien plus tôt chez Berenice Abbott, avec par exemple son projet Changing New York.

\section{ci-contre}

fig. 4

Détails architecturaux: porte d'entrée et balustrade à moitié détruites. Photos Mosbah Assi. Avec l'aimable autorisation de Solidere. 
12. Avec pour le premier la série Civil War (1977-1986) et pour la dernière le livre de 1984 (Ristelhueber 1984)

13. Elle donna lieu, notamment, à une exposition en 1992 au Palais de Tokyo à Paris, et des photographies furent éditées dans un ouvrage aujourd'hui épuisé (Eddé [dir.] 1992). Voir aussi le court métrage réalisé pendant la mission (Musso 1995).

14. Propos recueillis au cours d'un entretien avec Dominique Eddé à Paris, mars 2011.
Dominique Eddé, écrivain et éditorialiste libanaise, commanditaire de la mission de 1991, l'avait pilotée, avec la collaboration d'un directeur artistique qui n'était autre que Robert Delpire, fondateur du Centre national de la photographie (Paris) et éditeur des plus grands photographes, et celle de Bernard Latarget, qui avait codirigé en France de 1983 à 1989 avec François Hers la mission de la Délégation à l'aménagement du territoire et à l'action régionale (Datar). L'ambition de cette mission de la Datar avait été de proposer la réinvention du paysage français à travers la photographie (Hers et Latarget 1989; Bertho 2008; Guigueno 2006). Pour Depardon et Basilico, qui y avaient également pris part, elle avait marqué un moment important de leur carrière professionnelle, celui de l'affirmation d'un regard documentaire visant à produire une vision inédite ou du moins renouvelée de leurs sujets photographiques (la ferme du Garret où Depardon avait grandi, et le littoral français pour Basilico).

La Mission photographique de Beyrouth de 1991 avait quant à elle au Liban un caractère inédit. Elle eut un fort retentissement local et international. Les photographies qui en sont issues, celles d'un centre-ville suspendu entre la fin de la guerre et le début des travaux de reconstruction, allaient en effet marquer durablement les imaginaires associés à la ville. Leur esthétique documentaire renouvelait en effet les représentations de Beyrouth restées jusqu'alors dominées par la surabondance des photographies de reportage produites pendant la guerre. Si Raymond Depardon, Fouad Elkoury ou Sophie Ristelhuber (qui inaugurait là un long travail au MoyenOrient) avaient déjà produit des travaux préfigurant cette façon de traiter la ruine ${ }^{\mathbf{1 2}}$, ces productions étaient restées isolées. La nouveauté d'une mission collective, la reconnaissance dont jouissaient ses principaux organisateurs et la notoriété des photographes impliqués permirent de diffuser à l'échelle internationale une image renouvelée du Liban ${ }^{\mathbf{1 3}}$.

II s'agissait de construire une représentation de la destruction susceptible de résonner avec d'autres territoires en conflit. Financée par la Fondation Hariri alors que la société foncière Solidere n'avait pas encore vu le jour, la Mission photographique de Beyrouth entendait, pour reprendre la formule de sa commanditaire (Eddé 1992: 3), "témoigner du désastre» sans pour autant en donner directement à voir la dimension politique. Les photographes avaient donc pour consigne d'éviter de photographier toute figure humaine et tout signe partisan ${ }^{\mathbf{1 4}}$ (à l'époque déjà, des affiches, des pochoirs et des graffitis couvraient les murs de la capitale). Photographier des réfugiés ou des militaires, principaux usagers du centre-ville d'alors, aurait aussi pu faire croire à une photographie sociale dénonçant la misère d'une partie des Beyrouthins de l'après-guerre et éloigner de l'idée plus abstraite de la destruction de l'urbanité.

Pourtant, ce dessein ne rendit pas moins intense l'évocation photographique des conflits et de la violence. Les photographies de Basilico et Depardon adoptent une forme de neutralité que le premier, architecte de formation, atteint par une "photographie lente» excluant le mouvement et privilégiant la structure urbaine, et le second, par ce qu'il appelle la représentation des "temps morts", ces moments où rien apparemment ne se passe. Les ruines sont rendues plus abstraites chez Koudelka, qui se livre, dans des formats souvent panoramiques, à une exploration de la matière et de ses 


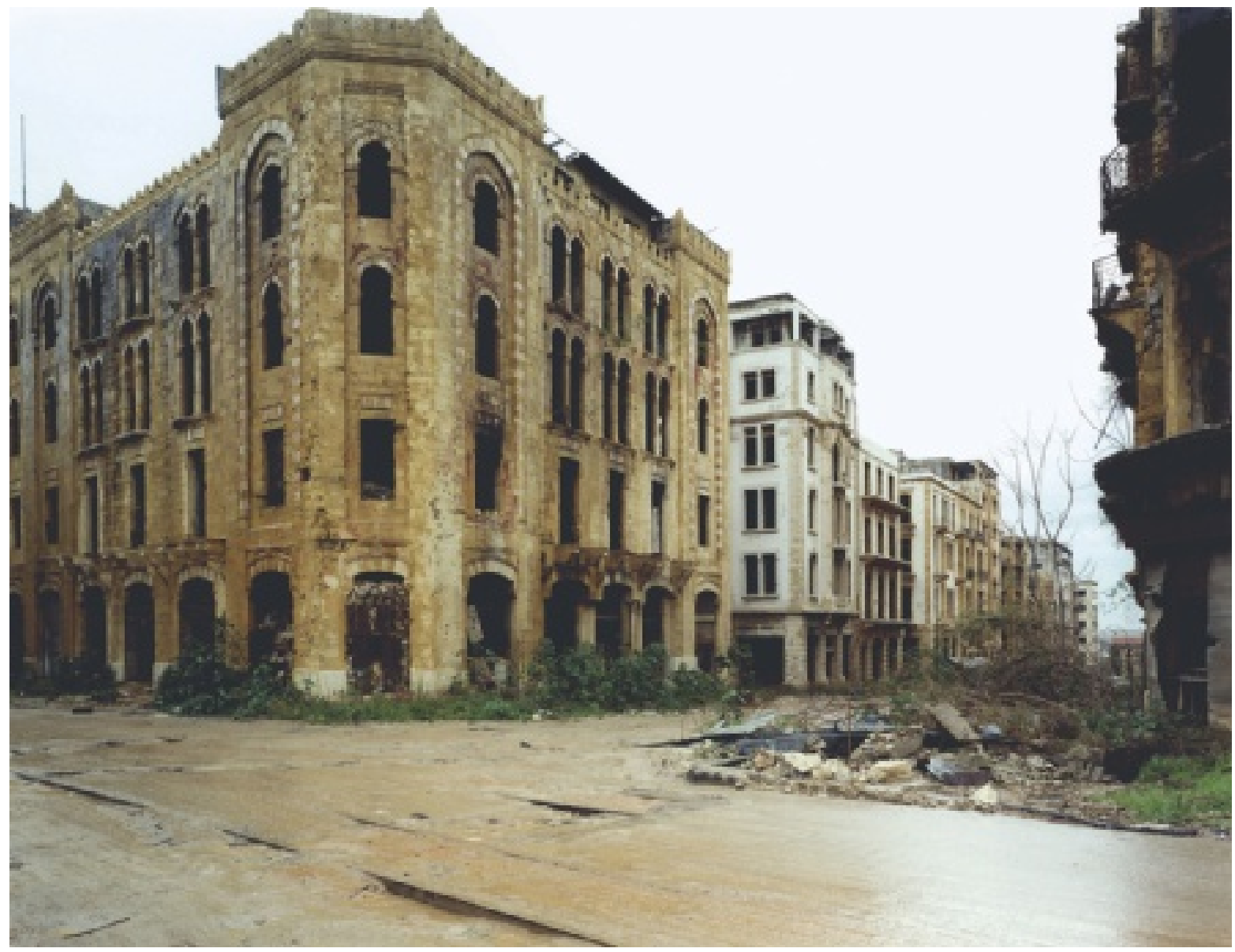

fig. 5

Gabriele Basilico,

Beyrouth, 1991

(c) Gabriele Basilico. 


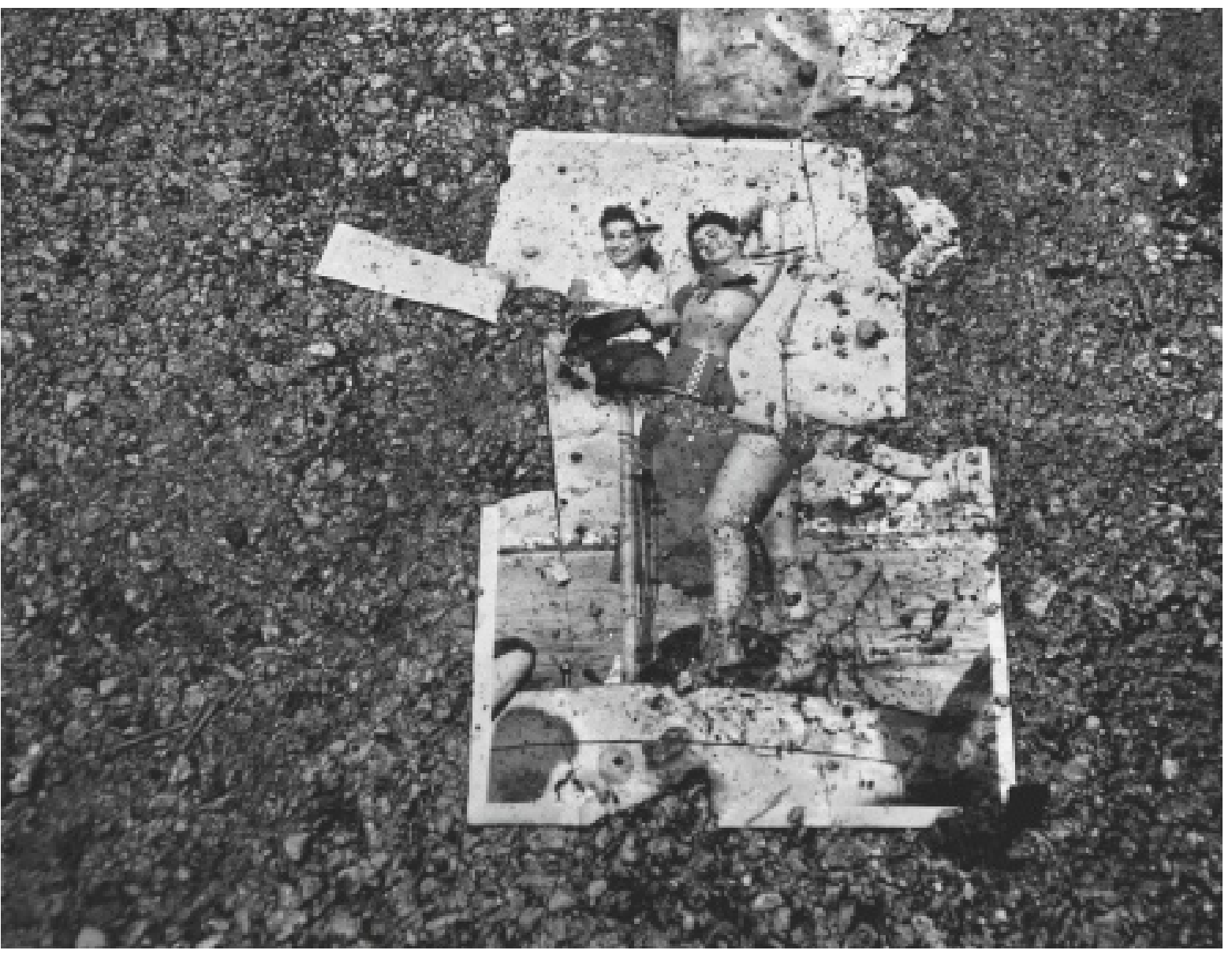

fig. 6

Fouad Elkoury, Beyrouth. Image sur asphalte, 1991 (C) Fouad Elkoury. distorsions brutales accentuées par un usage très contrasté du noir et blanc. Frank et Elkoury proposent, quant à eux, une lecture subjective et intime de la ville relevant toutefois de points de vue différents: le premier, adepte du montage, travaille ici l'écriture photographique du paysage et la mise en abyme, là où le second photographie une ville qu'il connaît fort bien, tant à travers son expérience quotidienne que dans une pratique photographique déjà ancienne (Basilico 2004; Depardon 2010; Elkoury 1984). En dépit de ces différentes approches, les photographies de la mission de 1991 érigent la ruine au rang de catégorie esthétique. En cela, la mission préfigure l'intérêt, dans les années 1990, des artistes et photographes locaux pour la question des «traces", des archives, du travail de mémoire, et leur affirmation de la nécessité de réinvestir un espace public menacé par la mainmise d'une entreprise privée sur l'ancien centre-ville, en dénonçant une forme d'amnésie collective entretenue par l'absence de justice et la reconversion des anciens chefs de guerre en leaders politiques.

Au-delà de cette ambition commune visant la réinvention de l'objet photographié à travers l'écriture documentaire, la mission de la Datar et celle de 1991 se rejoignaient également dans leur dimension patrimoniale. En effet le projet de la Datar avait lui-même été défini en référence à la Mission héliographique, première grande commande publique passée en 1851 à des photographes 
chargés de dresser pour la Commission des monuments historiques un inventaire photographique des principaux sites et monuments antiques et médiévaux de France, en vue de leur étude et de leur éventuelle restauration (Mondenard 1997). Pour la Datar, il s'agissait aussi, dans le contexte d'une politique de décentralisation, de faire un état des lieux du paysage français afin de décider de la possibilité d'entreprendre des aménagements significatifs. À Beyrouth, la guerre venait de prendre fin, et le quartier, en ruine, rendait d'autant plus urgente la question patrimoniale.

Ce tableau présente l'implication des photographes dans les différentes missions et entreprises photographiques indépendantes citées:

\begin{tabular}{|c|c|c|c|}
\hline Missions & Dates & Lieux concernés & Photographes \\
\hline Datar & 1983-1989 & France entière & $\begin{array}{l}\text { Sophie Ristelhueber, Josef Koudelka, } \\
\text { Raymond Depardon, Gabriele Basilico } \\
\text { ainsi que } 25 \text { autres photographes } \\
\text { français et étrangers: } \\
\text { Dominique Auerbacher, Lewis Baltz, } \\
\text { Bernard Birsinger, Alain Ceccaroli, Marc } \\
\text { Deneyer, François Despatin et Christian } \\
\text { Gobeli, Robert Doisneau, Tom Drahos, } \\
\text { Philippe Dufour, Gillbert Fastenaekens, } \\
\text { Pierre de Fenoÿl, Jean-Louis Garnell, } \\
\text { Albert Giordan, Franck Gohlke, Yves } \\
\text { Guillot, Werner Hannapel, François Hers, } \\
\text { Suzanne Lafont, Christian Meynen, } \\
\text { Christian Milovanoff, Vincent Monthiers, } \\
\text { Richard Pare, Hervé Rabot, Holger } \\
\text { Trülzsch }\end{array}$ \\
\hline $\begin{array}{l}\text { Travail } \\
\text { photographique } \\
\text { indépendant } \\
\text { (avant 1998) }\end{array}$ & 1984 & Beyrouth & Sophie Ristelhueber \\
\hline $\begin{array}{l}\text { Mission } \\
\text { photographique } \\
\text { de Beyrouth }\end{array}$ & 1991 & $\begin{array}{l}\text { Beyrouth } \\
\text { centre-ville }\end{array}$ & $\begin{array}{l}\text { Fouad Elkoury, Gabriele Basilico, } \\
\text { Raymond Depardon, Josef Koudelka, } \\
\text { René Burri, Robert Frank }\end{array}$ \\
\hline $\begin{array}{l}\text { Beyrouth } \\
\text { Central District } \\
\text { Photographic } \\
\text { Mission }\end{array}$ & $\begin{array}{l}\text { Projet formulé } \\
\text { en } 1998 \\
\text { Plusieurs } \\
\text { missions } \\
\text { depuis } 2009\end{array}$ & $\begin{array}{l}\text { Beyrouth } \\
\text { centre-ville }\end{array}$ & $\begin{array}{l}\text { Plusieurs photographes libanais } \\
\text { et étrangers, dont parmi les membres } \\
\text { d'anciennes missions: } \\
\text { Elkoury, Basilico, Depardon, Burri, ainsi } \\
\text { que Bassam Lahoud, Ahmad Azakir, } \\
\text { Mazen Jannoun, Roger Moukarzel, Ziyah } \\
\text { Gafic, Youmna Medlej, Bassam Lahoud, } \\
\text { Fares Jammal, David Xavier, Ferran } \\
\text { Quevedo, Raphaël Moneo, Fulvio Roiter, } \\
\text { Klavdij Sluban, Robert Polidori, Larry } \\
\text { McPherson, David Partner, } \\
\text { Lane Barden }\end{array}$ \\
\hline
\end{tabular}


15. Ces documents m'ont été communiqués par Fouad Elkoury au cours d'un entretien (juin 2014). Qu'il en soit ici vivement remercié. Je reste seule responsable des éventuelles erreurs contenues dans ce texte.

16. Ils m'expliquèrent au cours d'un entretien que des photographes réputés devaient garantir une archive de qualité, capable d'affirmer

l'importance du centreville tel que développé par Solidere.

\section{La Beirut Central District Photographic Mission: représenter le changement urbain}

Dans la lignée de la Mission photographique de 1991, le projet de la Beirut Central District Photographic Mission consistait en une série de nouvelles missions entreprises à partir de 2009 afin de documenter l'évolution des travaux de reconstruction. Pour le photographe Fouad Elkoury, celles-ci auraient été le moyen d'obtenir une autorisation officielle et prolongée, dont lui et ses collègues avaient besoin pour pouvoir photographier «librement» ce territoire urbain désormais propriété d'une société privée ${ }^{15}$. II estimait qu'il était urgent de saisir l'évolution de cet espace en transformation constante. À ce propos, dans le projet qu'il avait soumis à Solidere en 1998, il décrivait la façon dont en trois ans, entre 1994 et 1997, la quasi-totalité du quartier qu'il avait connu avait disparu. Peut-être désireux de partager son désarroi, le photographe y suggérait d'associer au regard photographique autant d'histoires et de récits sur la mémoire du centre-ville (il utilise le terme anglais de fictions). Ces visions subjectives seraient selon lui les mieux à même de valoriser le support documentaire (document, en anglais), d'en garantir la diffusion et la transmission auprès d'un plus large public. De leur côté, les archivistes du département des publications étaient conscients de l'importance, tant documentaire qu'esthétique, des travaux produits au cours de la mission de 1991, et avaient fait acheter par Solidere certains tirages. Soucieux de garantir le rayonnement du «nouveau » centreville, le département invita donc dès 2009 plusieurs photographes libanais et étrangers, de renommée internationale ${ }^{\mathbf{1 6}}$, à photographier ce quartier, parmi lesquels, hormis Fouad Elkoury, Gabriele Basilico, Larry E. McPherson, David Partner, Klavdij Sluban, Robert Polidori, Lane Barden, Raymond Depardon et René Burri.

Dans leurs travaux, c'est souvent l'image d'une ville vide qui prévaut, dans la lignée des missions évoquées précédemment mais aussi d'une certaine histoire de la photographie ayant pour objet le changement urbain. Pour des raisons d'abord essentiellement techniques, liées notamment à de longs temps de pause, la photographie de ville au XIX ${ }^{e}$ siècle est en effet celle d'une ville vide (Hawker 2013). Mais la focalisation sur le thème de la métamorphose a aussi donné lieu à des photographies d'où la figure humaine est le plus souvent absente: que l'on pense à d'importants corpus, comme ceux d'un Charles Marville sur les transformations parisiennes d'Haussmann, d'un Albert Schwartz à Berlin entre 1860 et 1914, d'un August Sander à Cologne, ou encore à ceux des photographes de la Société de photographie de l'Académie royale s'attachant aux vestiges du Vieux Londres. Les photos des missions de Beyrouth renvoient également à une autre production de la photographie documentaire $d u x x^{e}$ siècle, qui traite des traces de conflits sur le paysage urbain ou du déclin des sociétés industrielles (New Topographics, Sophie Ristelhueber, Paul Seawright et Simon Norfolk, Yves Marchand et Romain Meffre, etc.). Ces représentations de la ville vide s'éloignent de celles prévalant dans la presse ou la photographie vernaculaire. Elles affirment un style documentaire qui revendique une certaine neutralité face à des scènes jugées suffisamment éloquentes, tout en s'inscrivant dans la lignée d'œuvres plus anciennes, comme celles de Charles Marville ou d'Eugène Atget, dont les témoignages durables sur la transformation des villes ont été reconnus par leurs successeurs comme des travaux posant les bases d'une esthétique nouvelle de la photographie (Lugon 2001). Ces considérations n'apparurent pas 
cependant au cours de l'enquête auprès des archivistes de Solidere, lesquels envisagent plutôt la photographie comme étant partagée entre, d'un côté, une forme d'objectivisme, et de l'autre, une vision artistique. En effet, parmi les justifications invoquées pour ces missions, ils insistèrent davantage sur la notoriété des photographes et le gain de prestige pour la société foncière, que sur le souci de s'inscrire dans une quelconque tradition photographique. Et si leur discours reprend les arguments développés par Elkoury pour la Beirut Photographic Mission, il évolue aussi en fonction de leurs besoins et objectifs propres. Par exemple, là où le photographe affirmait l'importance de porter un regard d'auteur potentiellement critique sur l'évolution du quartier, les archivistes expliquent que les missions mobilisent des professionnels à la fois expérimentés et dont la subjectivité garantit la valeur artistique des photographies. Ce faisant, ils adhèrent à une pratique documentaire ayant intégré une dimension «artistique», reprenant à leur compte l'idée d'un documentaire de qualité, plus esthétique que purement descriptif (Lugon 2001: 29-30). Cette catégorie descriptive sert, quant à elle, à qualifier les photographies produites par le photographe salarié de l'autre département. Aussi, les archivistes du département des publications dotent-ils «leur» photographie d'une forme de supériorité sur la «documentation photographique " gérée par l'autre département, en valorisant sa dimension esthétique, artistique, en lieu et place de sa dimension potentiellement critique, et en instaurant, de ce fait, une ligne de partage entre ce qui appartiendrait d'une part au domaine de l'art - de la subjectivité - et, de l'autre, à celui de la neutralité scientifique (fig. 6).

\section{Discours sur la photographie:}

\section{l'archive et la politique culturelle}

Une photographie neutre ou «artistique "... Les publications de Solidere - brochures, rapports annuels - régulièrement diffusées auprès des actionnaires de la société foncière donnent corps à ces espaces discursifs distincts. Entre 2009 et 2013, et, plus particulièrement, depuis qu'ils émanent du département de design de Solidere, les "rapports », initialement consacrés aux bilans financiers de l'entreprise, sont conçus comme de véritables livres objets où la photographie et le design graphique tiennent une place très importante ${ }^{17}$. Ils reflètent une nouvelle dimension des activités de Solidere, qui cherche à s'imposer dans le paysage culturel local.

Le rapport de 2012, publié comme les autres en anglais, est intitulé The Chronicle. Il est constitué de deux livrets reliés à la suisse, qui s'ouvrent en vis-à-vis à l'intérieur d'une couverture rigide. Le livret de gauche est consacré au bilan financier de l'entreprise et à l'exposé de «faits et d'événements qui ont marqué la reconstruction et le développement du centre-ville" (Solidere 2012: page de couverture du livret de comptes). II y est écrit que "comme un calendrier, il évolue suivant la chronologie, sans analyse ni interprétation». Le livret de droite est intitulé: «Une série de photographies qui documentent la reconstruction et le développement du centre-ville de Beyrouth. » Un chapeau indique que «l'album photographique documente la régénération du centre de Beyrouth pendant vingt ans». II est "un témoin de la résurgence de la capitale libanaise, une précieuse archive d'histoire urbaine ». Ce livret présente une succession de photographies du fonds
17. Les rapports ont concouru et remporté plusieurs prix dans le domaine du design international, comme le Red Dot Design Award, I'If Design Award et le German Design Award (pour ceux de 2009 à 2011). double page suivante

Mazen Jannoun, Vue depuis le centre Starco, Minet El Hosn, 2011 (C) Mazen Jannoun. 
A.

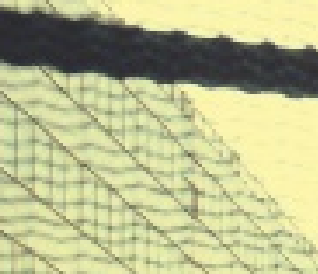

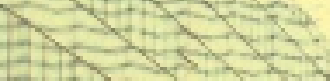

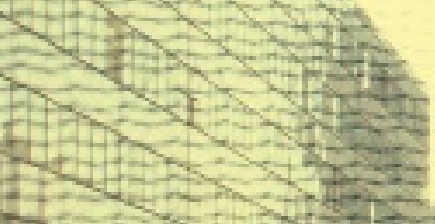

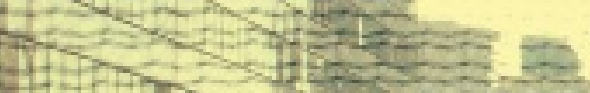

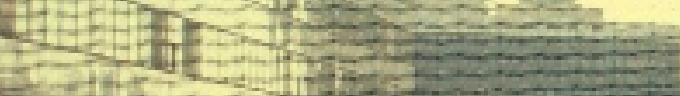

toplas

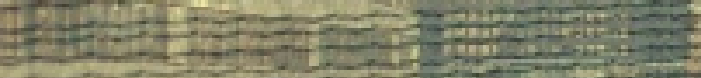

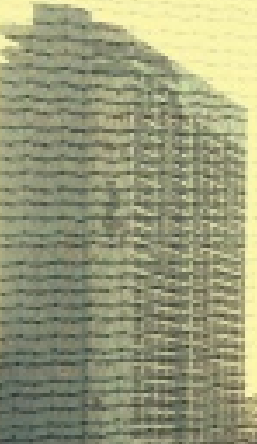

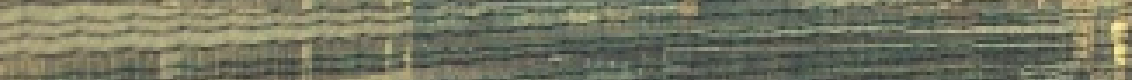

(x)

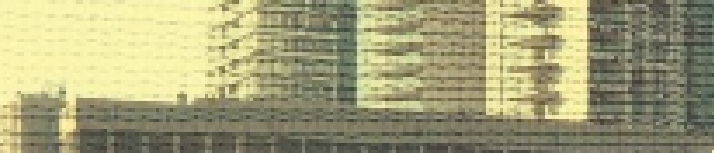

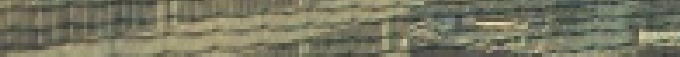

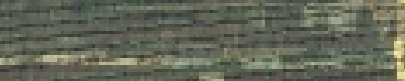

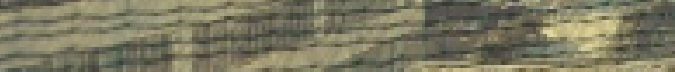

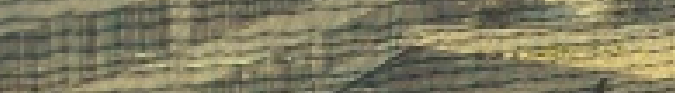

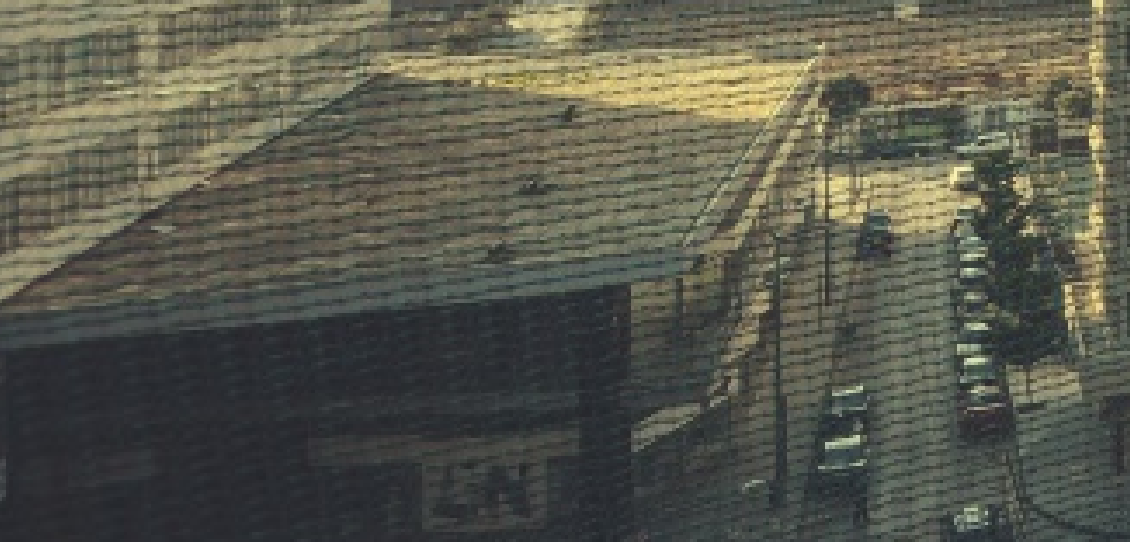

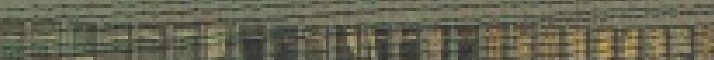

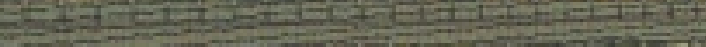

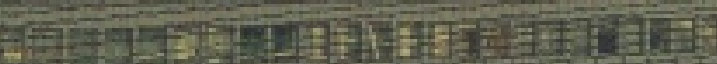

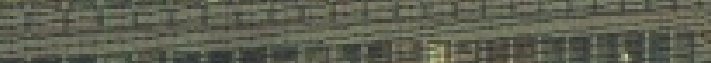

Adifind

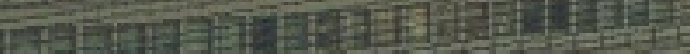

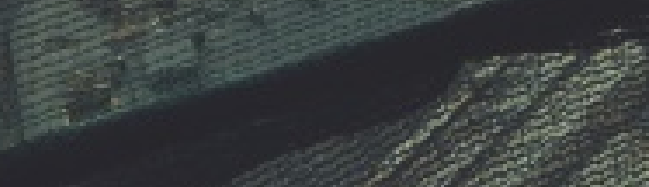




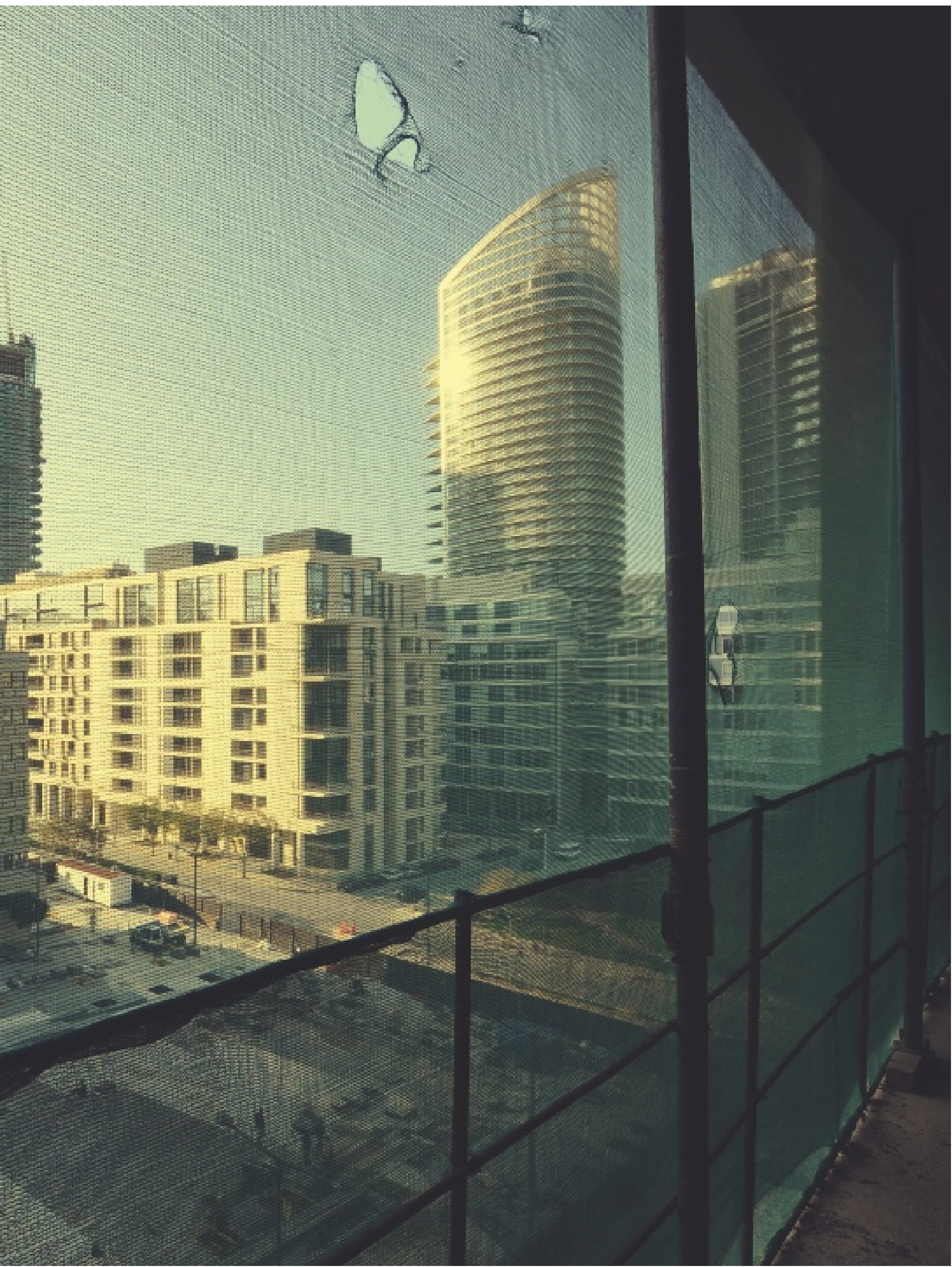




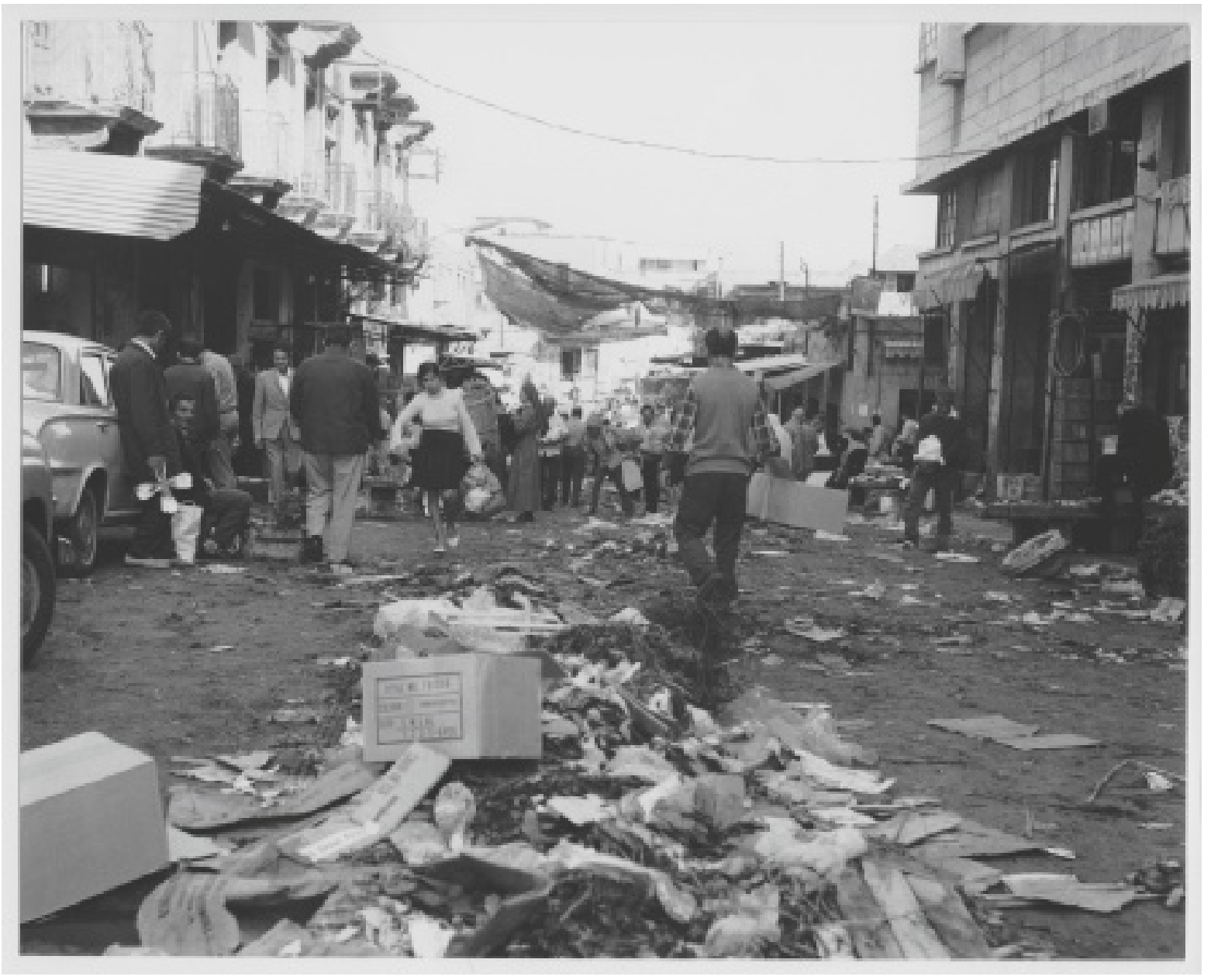

fig. 8

Photographie des souks

de Beyrouth publiée

dans le rapport annue

de Solidere 2012 (livret

de photographies).

Avec l'aimable autorisation

de Solidere. 
du département de communication, avec un hiatus entre 1991 et 1993, période où les démolitions de Solidere au centre-ville furent les plus intenses. Mais le photographe de Solidere n'y travaillait pas encore ${ }^{18}$. Avant 1994, ne sont montrées que les zones sauvegardées et restaurées par Solidere (alors que d'autres démolitions importantes eurent lieu jusqu'en 1998, notamment dans les secteurs de Wadi Abou Jmil, de Saïfi et du front de mer). Sont ensuite présentés des projets de développement et de constructions nouvelles. Relié du côté droit, ce livret invite à une lecture à double sens, de droite à gauche ou de gauche à droite. D'où l'attention particulière que j'ai portée aux deux photographies de début et de fin.

Sur la première (fig. 8) non légendée et datée de 1991, la vue d'un marché en noir et blanc occupe une demi-page. Le cadrage, avec une ligne d'horizon dans la partie supérieure de l'image, qui ne laisse qu'une place très réduite au ciel, accroît l'importance du premier plan: une rue en terre battue où stationnent des voitures, jonchée d'une épaisse couche de déchets en son centre et bordée, dans ce qui constituerait un second plan, par des immeubles d'époques diverses, dont les rez-de-chaussée sont occupés par des marchands de primeurs abrités sous des toits de fortune en tôle ondulée. L'angle choisi met l'accent sur la faible cohérence architecturale de cet espace, sa densité, son caractère sombre, enclavé et peu entretenu. D'autres photographies de l'époque donnent une image bien plus urbaine - à la fois plus structurée et vibrante - des marchés du centre-ville, alors que celle-ci insiste sur la désuétude du quartier, l'un des principaux arguments retenus par les urbanistes de Solidere pour justifier la démolition des anciens tissus du centre-ville. Retour à l'image: des bâches éventrées tendues des deux côtés de la rue brouillent la lecture de l'arrière-plan. L'une des rares figures vraiment lisible, parce que tournée vers nous, est cette femme qui apparaît en mouvement, au second plan, avec son pull-over blanc, parmi des hommes allant de dos. Son corps se courbe sous le poids d'un sac de courses. Le hors-champ temporel de la photographie s'immisce ici dans la lecture: en poursuivant sa marche, avec ses chaussures blanches assorties à son pull-over, elle devra bientôt éviter de piétiner les déchets qui jonchent le sol.

La dernière photographie du livret (fig. 9), en couleurs, occupe quant à elle une double page. On y voit le yacht-club en cours de construction dans la marina de Beyrouth. Côté sujet, nous passons du marché traditionnel hérité de l'époque ottomane à cet espace d'agrément des riches citadins, semblable à ceux d'autres métropoles maritimes internationales. La composition, également, contraste avec la première photographie. Ici, ciel et mer occupent une surface égale dans une l'image structurée par une forte horizontalité (la mer, l'horizon, l'architecture). L'espace, totalement ouvert, est habité par la nouvelle construction aux lignes contemporaines, signe de la modernité du quartier. L'usage de la couleur et d'un faible contraste renforce l'idée d'une atmosphère harmonieuse et une sensation de sérénité que traduit aussi la mer d'huile au premier plan. La légende renvoie aux qualités de l'image en proposant un surplus de sens: "La structure du yacht-club semble émerger naturellement du sol et transcende la frontière entre la ville et la mer. Le projet est un exemple emblématique de la richesse de la façade maritime».
18. En 1992 notamment une grande partie des anciens souks et des abords de la place des Martyrs fut démolie. 
19. Basilico est venu en mission en 1991, 2003 et 2011, à la demande, pour les deux dernières dates, du département des publications

20. Entretien avec le responsable des bases de données et des réseaux au département des publications, Solidere, janvier 2014.

21. Entretien avec l'une des archivistes du département des publications, Solidere, janvier 2014
Le choix de ces images et leur positionnement dans le livre jouent sur la transparence supposée des photographies pour livrer un message peu anodin : "Voilà comment étaient les marchés de Beyrouth avant la guerre ", et «voici, comme le montre le quartier modernisé par Solidere, la prospérité retrouvée». Dans le livret de gauche, le photographe Mosbah Assi décrit sur deux pages sa démarche dans l'élaboration de la documentation photographique (Assi 2012). À ses débuts, sa mission consistait, écrit-il, à « documenter tout ce qui se passait au centre-ville, l'état de chaque rue, entrée d'immeuble, balcon, élément de maçonnerie et fenêtre - au cas où cela nous serait utile au stade de la reconstruction " (ibid. : 10, trad. de l'auteure). Il ajoute: «L'objectif était de recourir à un enregistrement photographique de l'état global du centre-ville afin d'évaluer à l'avenir les efforts de reconstruction et de développement entrepris par Solidere. " (Ibid., trad. de l'auteure) La revendication d'une forme d'exhaustivité, de l'enregistrement systématique fondé sur l'utilisation d'un médium neutre, est ici à replacer dans le contexte non seulement de la stratégie éditoriale décrite plus haut, selon laquelle l'agencement des photographies dans le livre et le jeu sur leur contraste produit un effet de discours, mais aussi dans celui de l'histoire urbaine, celle d'une vive contestation du projet urbain de Solidere et des démolitions extensives entreprises avant 1993 (date d'embauche du photographe) [Brones 2010; Verdeil 2002]. Cet usage éditorial des photographies comme preuve d'une situation conforme à sa représentation (historique ou actuelle) pose clairement la question de l'ambivalence du savoir qu'elles délivrent. Comme cela est précisé, la documentation photographique devait accompagner un effort de reconstruction et de développement urbain. La campagne de photographies impliquait donc nécessairement une sélection des immeubles, tout comme le projet de reconstruction avait sélectionné, outre les édifices religieux, deux cent soixante-cinq immeubles sur la totalité des bâtiments encore debout après la guerre. L'absence quasi totale de discours critique sur la photographie, l'a priori de la neutralité du médium et de sa transparence permettent de fonder à partir des images un discours pacifié sur la ville et sa reconstruction.

Au contraire de ce rapport de 2012, celui de 2010 assume une totale subjectivité. Intitulé City in Layers, il s'articule autour du travail de Gabriele Basilico à Beyrouth. Y sont juxtaposées des photographies aux cadrages souvent équivalents, prises à plusieurs années d'intervalle, et des commentaires des images, où le photographe nous livre ses impressions sur les transformations du paysage et la photographie ${ }^{19}$. Comme l'indique la préface du rapport annuel, il s'agit à travers ce dispositif de documenter «le travail de Solidere au centre-ville de Beyrouth, un lieu où l'histoire, le présent et le futur émergent et évoluent de manière inattendue "(trad. de l'auteure). La renommée et le style documentaire de Basilico permettent aux archivistes d'assumer la dimension "artistique" de ce rapport, espace propice à l'expression des "émotions" de l'auteur ${ }^{20}$. Ce cas révèle pourtant la façon dont le statut de la photographie documentaire est constamment négocié. Un conflit entre les archivistes aurait en effet éclaté au moment de la publication, exprimant un désaccord sur le statut du médium et du rapport annuel face à l'enjeu du rayonnement de la société foncière ${ }^{21}$. Les archivistes du département des publications affirmaient qu'un rapport annuel avec des photographies 


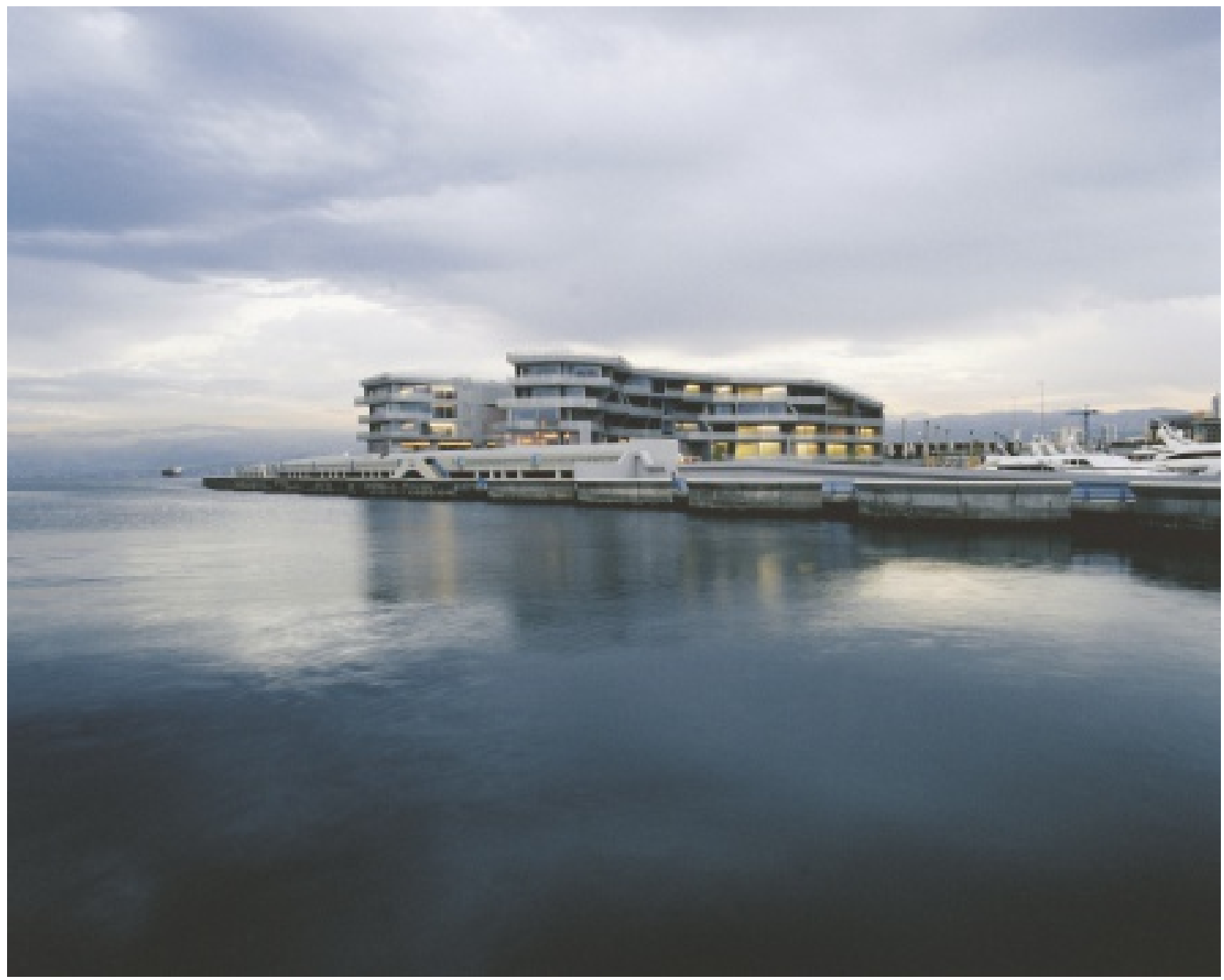

fig. 9

Photographie de la marina et du Yachtclub de Beyrouth publiée dans le rapport annuel de Solidere 2012 (livret de photographies).

Avec l'aimable autorisation de Solidere. 
22. Voir aussi son site Internet, qui présente en janvier 2018 la dernière publication, datée de 2014 http://www.portal9journal. org/index.aspx

23. Walker Evans, Porte, 204 West 13th Street, New York, vers 1931 ; Arnold Moses, Détail de porte, John Hazlet House, 2014 West 13th Street, New York, détail de la porte, 1936. éditées sur papier mat et une couverture souple en papier de Fabriano n'était pas digne d'accueillir les photographies d'un Gabriele Basilico, tandis que d'autres postulaient que, précisément, en privilégiant cette formule moins classique que celle d'une couverture rigide et d'un papier couché brillant, on rendrait plus accessibles des images d'une grande valeur documentaire et esthétique, en garantissant, dans le même temps, une meilleure diffusion aux publications de Solidere. Des critères esthétiques (des photographies de Basilico, mais aussi le choix du papier, de la texture, du design) et le travail d'un photographe mondialement reconnu offraient donc les bases d'une nouvelle identité éditoriale, développée depuis lors avec succès. II semblerait en effet que ce désaccord ait été, entre autres, à l'origine de la création du département de design (Solidere Multidisciplinary Design Team), en charge aujourd'hui non seulement de l'édition des rapports annuels, mais aussi d'essais d'auteurs libanais, publiés en anglais et arabe, ainsi que d'une revue critique, Portal 9, consacrée au Liban ${ }^{22}$. Cette production éditoriale qui mobilise des acteurs de la scène culturelle et littéraire permet à Solidere de s'inscrire dans le réseau des institutions culturelles locales tout en promouvant une image renouvelée de sa politique urbaine. Elle renforce aussi la distinction entre les deux départements et les deux catégories de photographies constituées par les archivistes. Pourtant, celles-ci se brouillent lorsqu'on regarde de près les objectifs et les méthodes de «documentation » mises en place par le photographe salarié de Solidere. En effet, étant donné que son travail photographique n'a été ni systématique ni toujours guidé par une recherche d'objectivité, et en considérant aussi ses photographies digitales, on peut finalement se demander où réside la différence avec le regard documentaire d'un photographe de renommée internationale, si ce n'est dans l'«espace discursif » (Krauss 1990a: 37) dans lequel elles sont mobilisées et par les itinéraires des images dans l'archive. C'est notamment une question que pose Olivier Lugon de façon très suggestive à propos de deux photographies d'une porte new-yorkaise, quasiment identiques, de Walker Evans et d'Arnold Moses, dont il écrit 23 : «Prises à quelques années d'intervalle, l'une a eu droit dès cette époque aux cimaises et aux portfolios du musée d'Art moderne, pendant que l'autre est immédiatement allée rejoindre les classeurs d'une société de défense du patrimoine - les archives de l'Historic American Buildings Survey, où elle jaunit depuis. Une telle injustice mérite, encore aujourd'hui, d'être interrogée. » (Lugon $2001: 38)$

Forte de ce pouvoir de détention de l'archive, Solidere s'efforce à travers ses publications de produire un discours sur l'histoire du quartier. Mais précisément, en mêlant deux registres de pratiques (archivage/écriture de l'histoire), en mélangeant les genres (rapport annuel/beau livre ou album photographique), elle fait l'économie de l'analyse critique des sources, qui caractérise pourtant le travail de l'historien. En opposant les valeurs d'objectivité et de travail artistique de la photographie, elle affirme d'un côté une histoire neutralisée du centre-ville et de l'autre la subjectivité et l'émotion de photographes auteurs face à ses transformations. Faire collection et maîtriser l'image par l'archive est donc pour la société foncière un moyen de renforcer son pouvoir symbolique en étant à la fois moteur d'une évolution urbaine, lieu de savoir et de mémoire. Pour autant, en archivant 
des photographies, qui plus est des images du changement urbain, Solidere ne peut prétendre effacer leur part de mystère. Celle-ci tient à leur existence même - des représentations de ce qui nécessairement n'est plus -, irréductible à des discours. Solidere ne fait alors que révéler davantage une impossibilité: celle d'utiliser la photographie comme un outil de pacification.

$$
\begin{array}{r}
\text { Ecole Nationale Supérieure d'Architecture de Versailles - LéAV } \\
\text { Institut Interdisciplinaire d'Anthropologie du Contemporain - } \\
\text { Laboratoire Anthropologie Urbanités Mondialisation } \\
\text { sophie.brones@versailles.archi.fr }
\end{array}
$$




\section{About, Ilsen et Chéroux, Clément}

2001 «L'histoire par la photographie", Études photographiques 10: 8-33.

\section{Assi, Mosbah}

2012 «On Photo Documenting the Reconstruction and Development of Beirut City Center ", in The Chronicle. Beyrouth, Solidere: 10-11.

\section{Barthes, Roland}

1980 La Chambre claire: note sur la photographie. Paris, Gallimard/Seuil.

\section{Basilico, Gabriele}

2004 Beyrouth 1991 (2003). Paris/Beirut, Le point du jour/ Solidere.

Bassil, Karl, Maasri, Zeina et Zaatari, Akram (avec Walid Raad)

2008 [2002] Mapping Sitting. On Portraiture and Photography. Beyrouth, Arab Image Foundation/ Mind the Gap.

\section{Bazin, André}

1999 [1945] «Ontologie de l'image photographique", in Qu'est-ce que le cinéma? Paris, Cerf: 9-17.

\section{Bertho, Raphaële}

2008 "Analyse de la genèse institutionnelle de la Mission photographique de la Datar» [en ligne], disponible sur: http:// halshs.archives-ouvertes.fr/ docs/00/71/58/25/PDF/AUX ORIGINES_MPD_ARTICLE.pdf (consulté le 5 avril 2018). Dans le cadre de la journée d'étude Établissement

de la photographie dans le paysage culturel français (1969-1981), INHA, 2008.

\section{Brones, Sophie}

2010 Beyrouth et ses ruines (1990-2010): une approche anthropologique. Thèse de doctorat, université Paris Ouest - Nanterre La Défense.

2014 "Le présent du patrimoine. Le décentrement des ayants droit dans la reconstruction de Beyrouth ", in Caroline de Saint-Pierre (dir.), La Ville patrimoine: formes, logiques, enjeux et stratégies. Rennes, Presses universitaires de Rennes: 39-56.

\author{
Depardon, Raymond \\ 2010 Beyrouth, centre-ville. \\ Paris, Points.
}

\section{Depaule, Jean-Charles}

2009 "La Fondation arabe pour l'image ", Vingtième Siècle 103 : 239-242.

À paraître "De quoi s'agit-il? ", in Christine Jungen et Jihane Sfeir (dir.), Archiver au Moyen-Orient. Paris, Karthala-IISMM

À paraître "Archiver des photographies au Proche-Orient: la Fondation arabe pour l'image". in Christine Jungen et Jihane Sfeir (dir.), Archiver au Moyen-Orient.

Paris, Karthala-IISMM.

Eddé, Dominique (dir.), photographies Gabriele Basilico, Raymond Depardon, Fouad Elkoury et al.

1992 Beyrouth centre ville. Paris, Éditions du cyprès.

\section{Elkoury, Fouad}

1984 Beyrouth aller-retour. Paris, Éditions de l'étoile.

\section{Guigueno, Vincent}

2006 "La France vue du sol. Une histoire de la mission photographique de la Datar (1983-1989) », Études photographiques 18: 96-119.

\section{Jungen, Christine et Raymond, Candice}

2012 "Introduction»,

Ateliers d'anthropologie 36.

\section{Hawker, Rosemary}

2013 «Repopulating the Street: Contemporary Photography and Urban Experience ", History of Photography 37 (3) : 341-352.

Hers, Bernard et Latarget, François (dir.), photographies de Holger Trülzsch, Sophie Ristelhueber, Raymond Depardon et al.

1989 Paysages, photographies: en France les années quatre-vingt Paris, Hazan/Datar/Crédit foncier de France.

\section{Krauss, Rosalind}

1990a «Les espaces

discursifs de la photographie",

in Le photographique: pour une théorie des écarts, trad. de l'anglais par Marc Bloch et Jean Kempf. Paris, Macula: 37-56.

1990b "Les noctambules ", in Le photographique: pour une théorie des écarts, trad. de l'anglais par Marc Bloch et Jean Kempf. Paris, Macula: 138-153.

\section{Lugon, Olivier}

2001 Le Style documentaire: d'August Sander à Walker Evans, 1920-1945. Paris, Macula.

\section{Makdissi, Nouhad (dir.), photographies Gabriel Basilico, Fouad Elkoury, Robert Polidori et Klavdij Sluban}

À paraître en 2018 Beirut Mission: photos 2009-2011. Göttingen/Beyrouth, Steidl/Solidere.

\section{McPherson, Larry E.}

2006 Beirut City Center. Göttingen, Steidl.

\section{Mondenard, Anne (de)}

1997 "La Mission héliographique: mythe et histoire ", Études photographiques 2.

\section{Piette, Albert}

1992 Le Mode mineur de la réalité: paradoxes et photographies en anthropologie. Louvain, Peeters.

\section{Ristelhueber, Sophie}

1984 Beyrouth, photographies. Paris, Hazan.

\section{Shore, Stephen}

2010 [2007] Leçon de photographie: la nature des photographies, trad. de l'anglais par Dominique Lablanche. Paris, Phaïdon.

\section{Solidere}

2012 The Chronicle, Annual Report Beyrouth, Solidere. Disponible en ligne sur: http://www.solidere.com/ sites/default/files/attached/ar2012. pdf (consulté le 5 avril 2018).

\section{Van Lier, Henri \\ 1983 Philosophie de la Les impressions nouvelles.}

\section{Verdeil, Éric}

Beyrouth en reconstruction.

Paris I Sorbonne.

\section{Filmographie}

\section{Musso, Tanino}

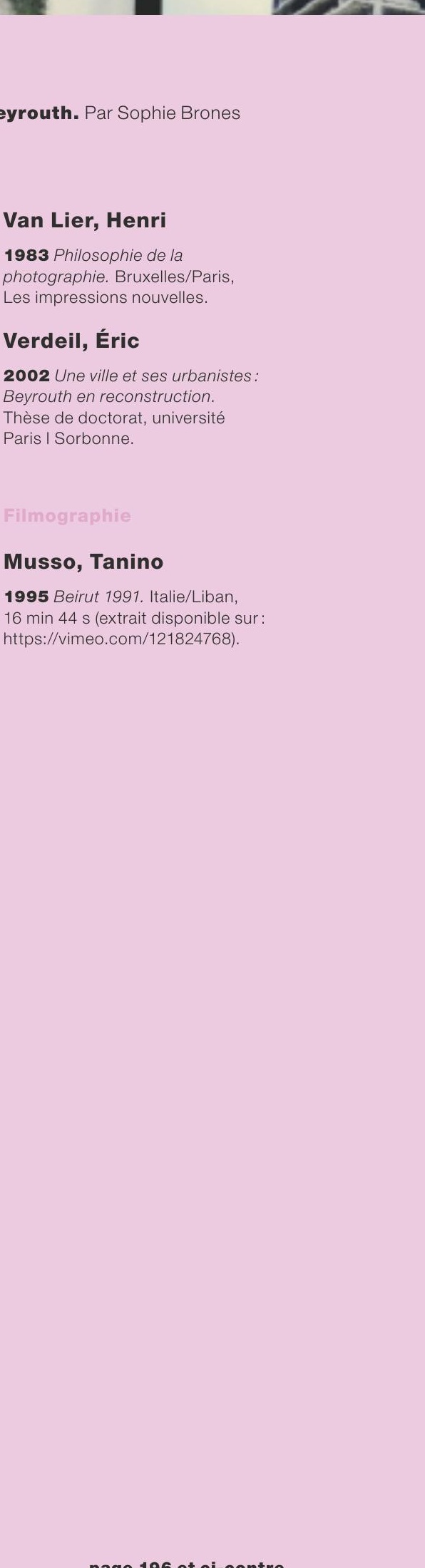

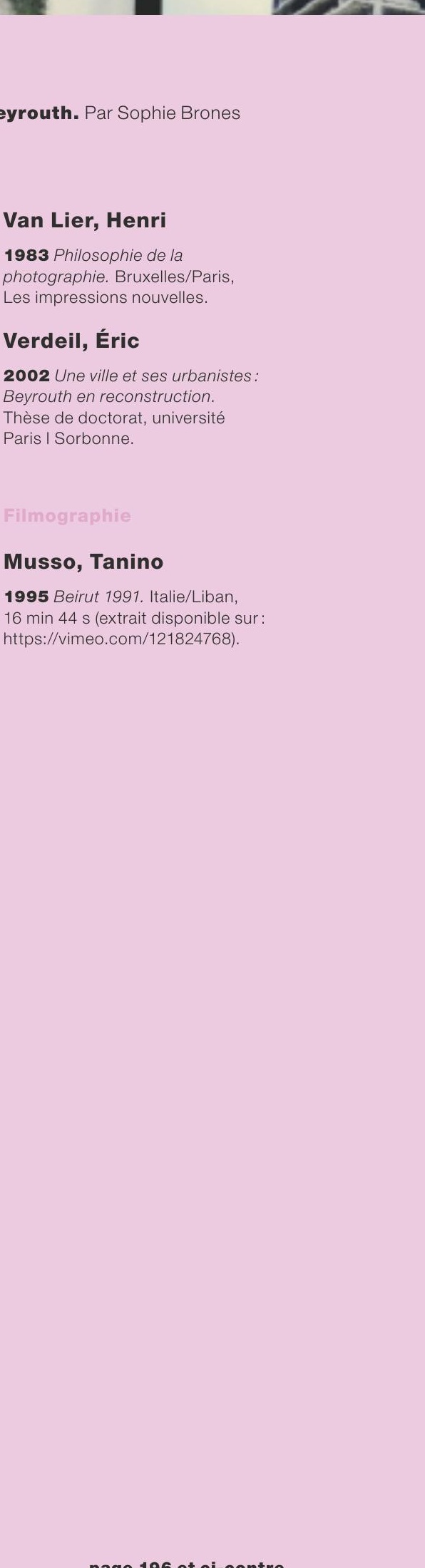

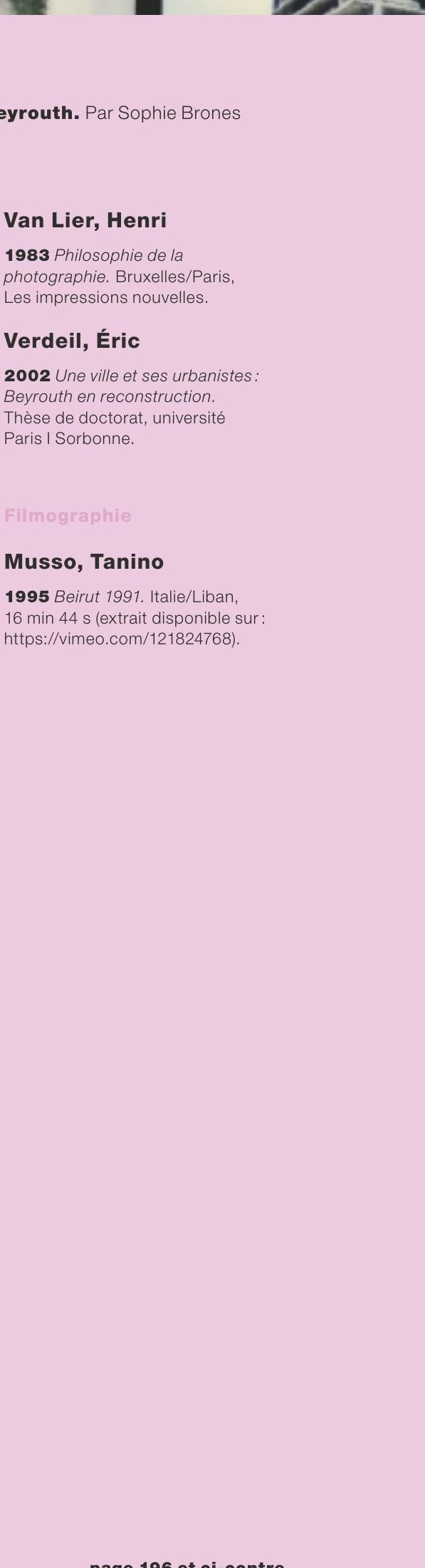

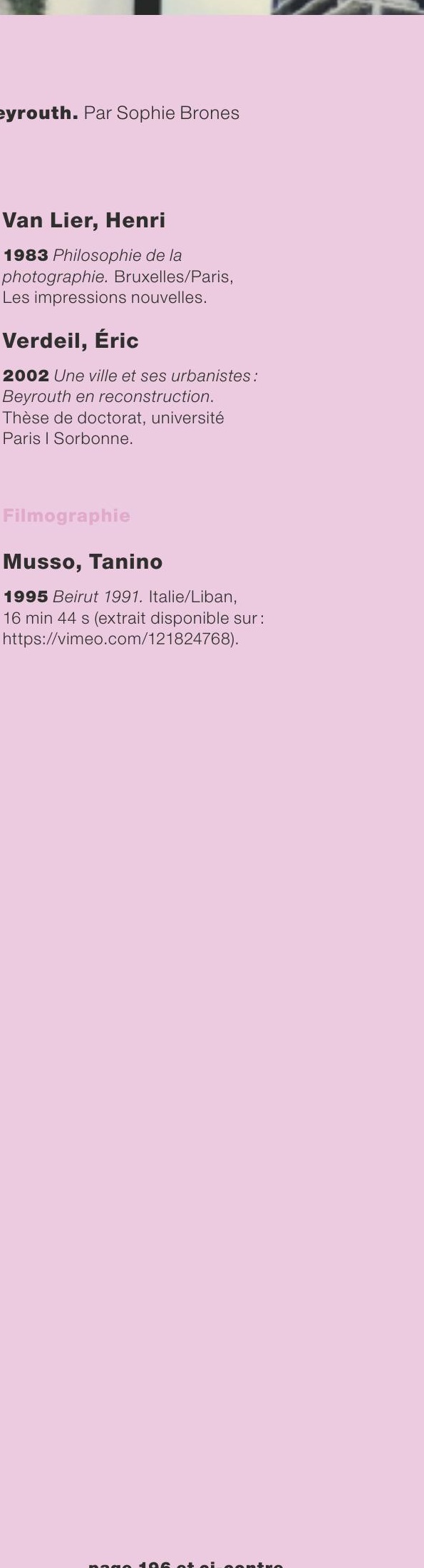

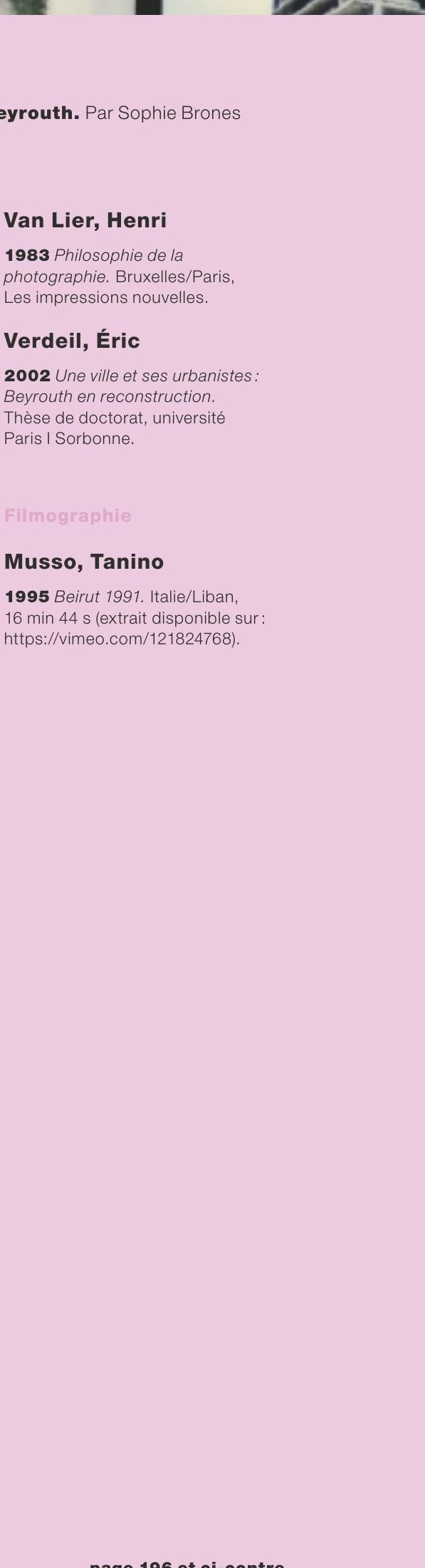

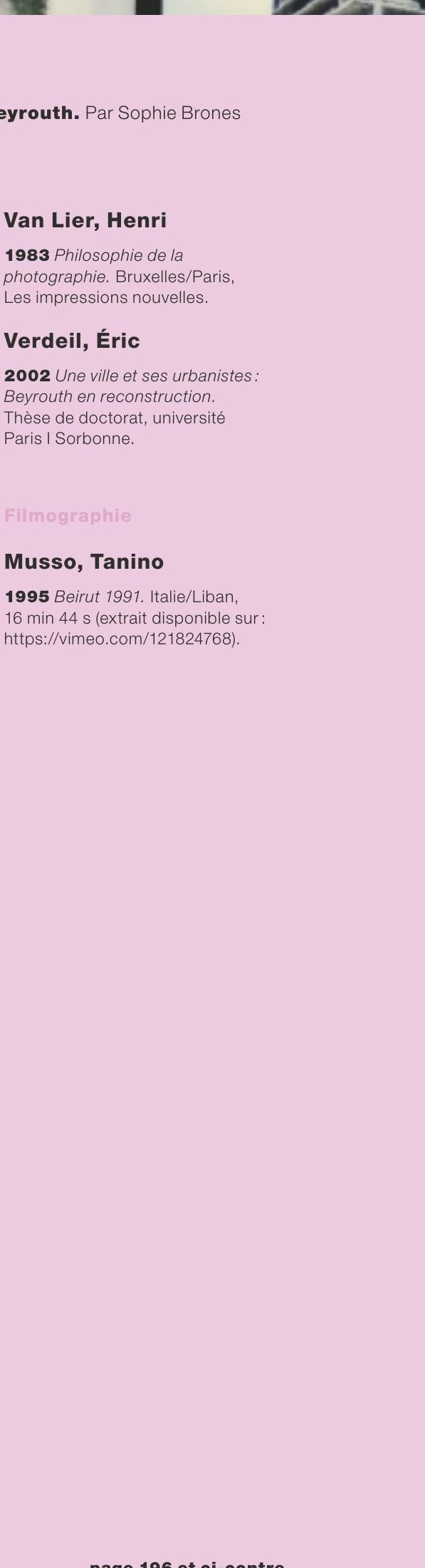

page 196 et ci-contre Exemple d'une planche-contact: série d'immeubles détruits au centre-ville. Photos Mosbah Assi. Avec l'aimable autorisation de Solidere. 
